LANDAU-97-TMP-5

hep-th/9710099

\title{
Free Field Construction for Correlation Functions of the Eight-Vertex Model
}

\author{
Michael Lashkevich and Yaroslav Pugai \\ L. D. Landau Institute for Theoretical Physics, \\ 142432 Chernogolovka, Russia
}

\begin{abstract}
A free field representation for the type $I$ vertex operators and the corner transfer matrices of the eight-vertex model is proposed. The construction uses the vertex-face correspondence, which makes it possible to express correlation functions of the eight-vertex model in terms of correlation functions of the SOS model with a nonlocal insertion. This new nonlocal insertion admits of a free field representation in terms of Lukyanov's screening operator. The spectrum of the corner transfer matrix and the Baxter-Kelland formula for the average staggered polarization have been reproduced.
\end{abstract}

October 1997

\section{Introduction}

The eight-vertex model ${ }^{1,2,3}$ is one of the most famous examples of exactly solvable models of statistical mechanics. Its partition function was found in 1971 by Baxter. ${ }^{4}$ Later expressions for the spontaneous polarization and magnetization were proposed. ${ }^{5,6}$ Nevertheless, very few results on correlation functions have been obtained up to now. . $^{3,8}$

For the last years, a new approach to calculating correlation functions of integrable (exactly solvable) lattice and continuous models has been developed. ${ }^{9-13}$ This approach, known as the vertex operator approach, has shown its efficiency in calculation and analysis of correlation functions and form factors for different integrable models, such as the six-vertex model, ${ }^{9-11}$ the restricted solid-on-solid (RSOS) models ${ }^{14-16}$ and their $A_{n}^{(1)}$ generalizations, ${ }^{17,18}$ the fusion RSOS models, ${ }^{19,20}$ the Ising model,${ }^{14,21}$ the sine-Gordon model. ${ }^{12,13}$ The most important constituent of this approach that allows one to perform calculations is the free field representation of the vertex operators.

However, the free field representation was not elaborated for the eight-vertex model. Formally, the problem lies in the presence of the $d$ matrix element in the $R$ matrix of this model which breaks down the charge conservation, which is necessary for the free field representation. In principle, the same reason gives no way of applying directly the Bethe ansatz method to this model. ${ }^{3}$ Baxter's idea to overcome this difficulty for the Bethe ansatz was to twist somehow the model to restore the charge conservation law without change of eigenvalues of the transfer matrix. ${ }^{22}$ Such 'twisted' model was the solid-on-solid (SOS) model, which is a face type model. It is related to the eight-vertex model by the so-called vertex-face correspondence.

It is an old idea to use this vertex-face correspondence to relate correlation functions of the eightvertex model with ones of the SOS model, ${ }^{23}$ and then to apply the known free field representation for the vertex operators of the SOS model. The obstacle is non-local character of the vertex-face correspondence for correlation functions. In this paper we analyze this relation and propose a free field approach for the correlation functions of the eight-vertex model. As a check we find the spectrum of the corner transfer matrix of the eight-vertex model, and reproduce the Baxter-Kelland formula ${ }^{5}$ for the staggered spontaneous polarization in the antiferroelectric phase.

The paper is organized as follows. In Sec. 2 basic definitions and notations are given. In Sec. 3 we give a review of the vertex operator approach of both the eight-vertex and the SOS models. In Sec. 4 we 


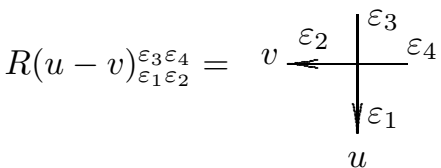

(a)

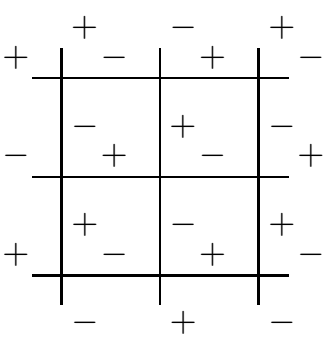

$(b)$

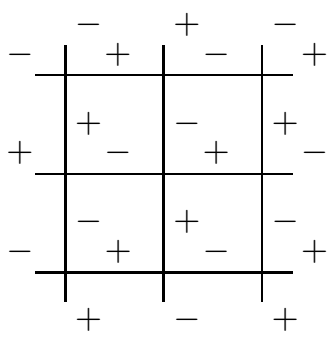

Fig. 1. Eight-vertex model: (a) definition of the weight matrix; (b) two degenerate ground states.

use the vertex-face correspondence to find a relation between correlation function of the eight-vertex model and the SOS model. The construction contains a new object in addition to corner transfer matrices and vertex operators. This object (operator $\Lambda$ in our designations) relates the corner transfer matrices of the eight-vertex model to those of the SOS model. In Sec. 5 we describe a free field representation and calculate the staggered polarization by use of the free field representation. In Sec. 6 we discuss main conjectures used in the paper and possible directions of future studies.

\section{Basic Definitions and Vertex-Face Correspondence}

\subsection{Eight-Vertex Model}

The eight-vertex model is defined as follows. ${ }^{3}$ The fluctuating variables (polarizations) $\varepsilon= \pm 1 \equiv \pm$ are placed at links of a square lattice. Interaction is associated with vertices of the lattice. A local weight $R_{\varepsilon_{1} \varepsilon_{2}}^{\varepsilon_{3} \varepsilon_{4}}$ is associated to each configuration of polarizations $\varepsilon_{1}, \varepsilon_{2}, \varepsilon_{3}, \varepsilon_{4}$ around a vertex (Fig. 1a). A weight of a lattice configuration is the product of local weights.

It is convenient to attach a constant complex 'spectral parameter' to each line of the lattice, and to consider a weight as a function of the difference of the spectral parameters of the two lines that intersect at the vertex. The weights as functions of this difference are given by ${ }^{3}$

$$
\begin{aligned}
& a(u)=R(u)_{++}^{++}=R(u)_{--}^{--}=-\mathrm{i} \kappa(u) R_{0}(u) \theta_{4}\left(\mathrm{i} \frac{\epsilon}{\pi} ; \mathrm{i} \frac{2 \epsilon r}{\pi}\right) \theta_{4}\left(\mathrm{i} \frac{\epsilon}{\pi} u ; \mathrm{i} \frac{2 \epsilon r}{\pi}\right) \theta_{1}\left(\mathrm{i} \frac{\epsilon}{\pi}(1-u) ; \mathrm{i} \frac{2 \epsilon r}{\pi}\right), \\
& b(u)=R(u)_{+-}^{+-}=R(u)_{-+}^{-+}=-\mathrm{i} \kappa(u) R_{0}(u) \theta_{4}\left(\mathrm{i} \frac{\epsilon}{\pi} ; \mathrm{i} \frac{2 \epsilon r}{\pi}\right) \theta_{1}\left(\mathrm{i} \frac{\epsilon}{\pi} u ; \mathrm{i} \frac{2 \epsilon r}{\pi}\right) \theta_{4}\left(\mathrm{i} \frac{\epsilon}{\pi}(1-u) ; \mathrm{i} \frac{2 \epsilon r}{\pi}\right), \\
& c(u)=R(u)_{+-}^{-+}=R(u)_{-+}^{+-}=-\mathrm{i} \kappa(u) R_{0}(u) \theta_{1}\left(\mathrm{i} \frac{\epsilon}{\pi} ; \mathrm{i} \frac{2 \epsilon r}{\pi}\right) \theta_{4}\left(\mathrm{i} \frac{\epsilon}{\pi} u ; \mathrm{i} \frac{2 \epsilon r}{\pi}\right) \theta_{4}\left(\mathrm{i} \frac{\epsilon}{\pi}(1-u) ; \mathrm{i} \frac{2 \epsilon r}{\pi}\right), \\
& d(u)=R(u)_{++}^{--}=R(u)_{--}^{++}=-\mathrm{i} \kappa(u) R_{0}(u) \theta_{1}\left(\mathrm{i} \frac{\epsilon}{\pi} ; \mathrm{i} \frac{2 \epsilon r}{\pi}\right) \theta_{1}\left(\mathrm{i} \frac{\epsilon}{\pi} u ; \mathrm{i} \frac{2 \epsilon r}{\pi}\right) \theta_{1}\left(\mathrm{i} \frac{\epsilon}{\pi}(1-u) ; \mathrm{i} \frac{2 \epsilon r}{\pi}\right)
\end{aligned}
$$

with $\theta_{i}(u ; \tau)$ is the $i$ th theta function with the basic periods 1 and $\tau(\operatorname{Im} \tau>0)$. Here $\epsilon$ and $r$ are parameters. It is often convenient to use another set of parameters

$$
x=\mathrm{e}^{-\epsilon}, \quad p=x^{2 r}, \quad z=x^{2 u} .
$$

We shall use both 'additive' parameters $u, \epsilon, r$ and 'multiplicative' parameters $z, x, p$ on equal grounds.

The common factor $\kappa(u) R_{0}(u)$ is chosen so that the partition function per site is equal to one:

$$
\begin{gathered}
\kappa(u)=z^{-\frac{r-1}{2 r}} x^{1-\frac{r}{2}}\left(x^{2 r} ; x^{2 r}\right)_{\infty}^{-2}\left(x^{4 r} ; x^{4 r}\right)_{\infty}^{-1}\left(x^{2} z^{-1} ; x^{2 r}\right)_{\infty}^{-1}\left(x^{2 r-2} z ; x^{2 r}\right)_{\infty}^{-1}, \\
R_{0}(u)=z^{\frac{r-1}{2 r}} \frac{\left(x^{2} z^{-1} ; x^{4}, x^{2 r}\right)_{\infty}\left(x^{2 r+2} z^{-1} ; x^{4}, x^{2 r}\right)_{\infty}\left(x^{4} z ; x^{4}, x^{2 r}\right)_{\infty}\left(x^{2 r} z ; x^{4}, x^{2 r}\right)_{\infty}}{\left(x^{4} z^{-1} ; x^{4}, x^{2 r}\right)_{\infty}\left(x^{2 r} z^{-1} ; x^{4}, x^{2 r}\right)_{\infty}\left(x^{2} z ; x^{4}, x^{2 r}\right)_{\infty}\left(x^{2 r+2} z ; x^{4}, x^{2 r}\right)_{\infty}} \\
\left(z ; p_{1}, \ldots, p_{N}\right)=\prod_{n_{1}, \ldots, n_{N}=0}^{\infty}\left(1-z p_{1}^{n_{1}} \ldots p_{N}^{n_{N}}\right) .
\end{gathered}
$$

Splitting the common factor into two parts is convenient for later use. 
The matrix $R(u)$ satisfies the Yang-Baxter equation, the crossing and unitarity properties. ${ }^{3,8}$ This supplies commutativity of transfer matrices with different spectral parameters $u$ (but coincident $\epsilon$ and $r$ ) and integrability of the model.

For real parameters $\epsilon, r, u$ in the region

$$
\epsilon>0, \quad r>1, \quad-1<u<1 \quad \text { or } \quad a+|b|+|d|<c
$$

the model is in the antiferroelectric phase. In the 'low temperature' limit $\epsilon \rightarrow \infty$ (or $a,|b|,|d| \ll c)$ the system falls in one of two ground states (Fig. 1b). These two ground states correspond to the broken $\mathbf{Z}_{2}$ symmetry. At finite temperatures the symmetry remains broken in the whole region (2.3). The second order phase transition takes place at $\epsilon=0$. The weight $d_{A}$ in our definition is negative but it enters into any configuration in an even power in the antiferroelectric phase and its sign is inessential.

The aim of the present paper is to find a procedure for calculating correlation functions in the eightvertex model in the thermodynamic limit. In our normalization the partition function per site is equal to 1. So we can define the correlation function directly on the infinite lattice. Let us label the vertices of the lattice of finite size by two integer Cartesian coordinates $(x, y)$. The coordinates of polarizations $\varepsilon(x, y)$ are the coordinates of the centers of links $\left(x \in \mathbf{Z}+\frac{1}{2}, y \in \mathbf{Z}\right.$ for vertical links and $x \in \mathbf{Z}, y \in \mathbf{Z}+\frac{1}{2}$ for horizontal ones). The probability that polarizations at definite links $\left(x_{k}, y_{k}\right), k=1, \ldots, N$, take fixed values $\varepsilon_{k}$ is given by

$$
P_{\varepsilon_{1} \ldots \varepsilon_{N}}^{(i)}=\sum_{\substack{\{\varepsilon(x, y)\} \\ \varepsilon\left(x_{k}, y_{k}\right)=\varepsilon_{k}}} \prod_{x, y \in \mathbf{Z}} R\left(u_{x}-v_{y}\right)_{\varepsilon\left(x, y-\frac{1}{2}\right) \varepsilon\left(x-\frac{1}{2}, y\right)}^{\varepsilon\left(x, y+\frac{1}{2}\right) \varepsilon\left(x+\frac{1}{2}, y\right)} .
$$

Here the sum is taken over all configurations such that $\varepsilon_{x_{k}, y_{k}}=\varepsilon_{k}$ and $\varepsilon_{x, y}$ only differ from the ground state configuration value $(-)^{x-y-\frac{1}{2}+i}$ at a finite number of links. Note, that we consider an inhomogeneous lattice, where each line brings its own spectral parameter $u_{x}$ of $v_{y}$, so that $u_{x}=u=$ const and $v_{y}=v=$ const for large enough $x$ and $y$ respectively. Later we concentrate our attention on the particular case of $N$ parallel neighboring links $\left(x_{k}=\frac{1}{2}, y_{k}=k\right.$, see Fig. 5a), but our consideration is valid in general case.

\subsection{SOS model}

Let us turn to the SOS model. Here one associates an integer variable ('height') $n$ to each site of a square lattice, so that the heights $n_{1}$ and $n_{2}$ at adjacent sites satisfy the admissibility condition

$$
\left|n_{1}-n_{2}\right|=1 \text {. }
$$

Interaction is described by weights $W\left[\begin{array}{ll}n_{4} & n_{3} \\ n_{1} & n_{2}\end{array} \mid u\right]$ associated to faces, and the spectral parameters are attached to lines of the dual lattice (Fig. 2):

$$
\begin{aligned}
W\left[\begin{array}{cc}
n & n \pm 1 \\
n \pm 1 & n \pm 2
\end{array} \mid u\right] & =R_{0}(u) \\
W\left[\begin{array}{cc}
n & n \pm 1 \\
n \pm 1 & n
\end{array} \mid u\right] & =R_{0}(u) \frac{[n \pm u][1]}{[n][1-u]} \\
W\left[\begin{array}{cc}
n & n \pm 1 \\
n \mp 1 & n
\end{array} \mid u\right] & =-R_{0}(u) \frac{[n \pm 1][u]}{[n][1-u]} .
\end{aligned}
$$

Here we use the designation

$$
\begin{gathered}
{[u]=x^{\frac{u^{2}}{r}-u} \Theta_{x^{2 r}}\left(x^{2 u}\right)=\sqrt{\frac{\pi}{\epsilon r}} \mathrm{e}^{\frac{1}{4} \epsilon r} \theta_{1}\left(\frac{u}{r} ; \frac{\mathrm{i} \pi}{\epsilon r}\right),} \\
\Theta_{p}(z)=(z ; p)_{\infty}\left(p z^{-1} ; p\right)_{\infty}(p ; p)_{\infty} .
\end{gathered}
$$

Note that the weights $W$ contain $[n]$ in the denominators. So the construction demands some regularization. Namely, we may think that the heights $n$ belong to the set $\mathbf{Z}+\delta$ with some real $\delta$. Such shift does not 


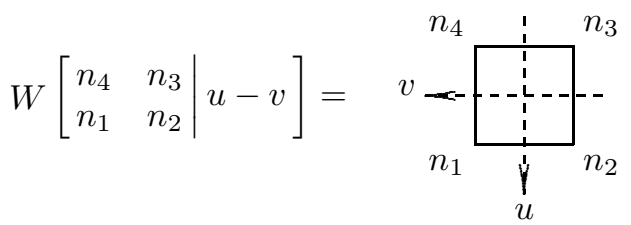



(b)

Fig. 2. SOS model: $(a)$ definition of weights; $(b)$ an infinite number of degenerate ground states labeled by an integer $m$ and by $s= \pm 1$ satisfying $(k-1) r<m, m+s<k r$ for some integer $k$.

break down the admissibility condition (2.5). After calculation of all physical quantities one may take the limit $\delta \rightarrow 0$. The consideration below is valid literally for the regularized case, and we shall imply this regularization everywhere. Moreover, the final results do not depend on $\delta$ at all.

The weights (2.6) satisfy a face version of the Yang-Baxter equation, the crossing and unitarity properties. ${ }^{22,14}$ Note that the elements (2.6a) are analogues of the element $a(u)$ of the eight-vertex model, (2.6b) are analogues of $c(u)$, and (2.6c) of $b(u)$. Namely, we can associate a sign variable $\varepsilon$ to each link of the dual lattice intersecting a link of the direct lattice between $n+\varepsilon$ and $n(n+\varepsilon$ is to the left or upper than $n)$. It is impossible to imagine any analog of $d(u)$ in this picture. The charge defined as the sum of the variables $\varepsilon$ along an infinite line consisting of links of the direct lattice is conserved by definition.

We are interested in the so called regime $I I I$ :

$$
\epsilon>0, \quad r \geq 1, \quad 0<u<1 \text {. }
$$

There is an infinite number of degenerate, but inequivalent, ground states in this regime shown in Fig. $2 \mathrm{~b}$.

\subsection{Vertex-Face Correspondence}

Consider the functions (Fig. 3a)

$$
t_{\varepsilon}(u)_{n}^{n^{\prime}}=\frac{\varepsilon^{n-m}}{\sqrt{2}}\left(\theta_{2}\left(\frac{\left(n^{\prime}-n\right) u+n^{\prime}}{r} ; \mathrm{i} \frac{2 \pi}{\epsilon r}\right)+\varepsilon \theta_{3}\left(\frac{\left(n^{\prime}-n\right) u+n^{\prime}}{r} ; \mathrm{i} \frac{2 \pi}{\epsilon r}\right)\right)
$$

with $m$ being an arbitrary integer (in the regularized version $m \in \mathbf{Z}+\delta$ ), $\varepsilon= \pm$ being a polarization variable, $n$ and $n^{\prime}$ being an admissible pair of heights $\left(i\right.$. e. $\left.\left|n^{\prime}-n\right|=1\right)$. These functions enter into the identity ${ }^{22}$ (Fig. 4a)

$$
\sum_{\varepsilon_{1}^{\prime} \varepsilon_{2}^{\prime}} R(u-v)_{\varepsilon_{1} \varepsilon_{2}}^{\varepsilon_{1}^{\prime} \varepsilon_{2}^{\prime}} t_{\varepsilon_{1}^{\prime}}\left(u_{0}-u\right)_{s^{\prime}}^{n^{\prime}} t_{\varepsilon_{2}^{\prime}}\left(u_{0}-v\right)_{n}^{s^{\prime}}=\sum_{s \in \mathbf{Z}} t_{\varepsilon_{2}}\left(u_{0}-v\right)_{s}^{n^{\prime}} t_{\varepsilon_{1}}\left(u_{0}-u\right)_{n}^{s} W\left[\begin{array}{cc}
n^{\prime} & s^{\prime} \\
s & n
\end{array} \mid u-v\right]
$$

which is referred to as vertex-face correspondence. The functions $t_{\varepsilon}(u)_{n}^{n^{\prime}}$ are called intertwining vectors $($ the subscript $\varepsilon$ is often regarded as a vector index). Note that the identity holds for arbitrary value of $u_{0}$. The vertex-face correspondence is the basic relation for solving the eight-vertex model by Bethe ansatz. Below we apply it to find the free field representation. The intertwining vectors may be pulled through the lattice by use of the vertex-face correspondence. ${ }^{a}$

It is convenient to introduce a 'conjugate' and a 'primed' intertwining vectors (Fig. 3b)

$$
\sum_{\varepsilon} t_{\varepsilon}^{*}(u)_{n}^{n^{\prime}} t_{\varepsilon}(u)_{n^{\prime \prime}}^{n}=\delta_{n^{\prime} n^{\prime \prime}}, \quad \sum_{\varepsilon} t_{\varepsilon}^{*}(u)_{n^{\prime}}^{n} t_{\varepsilon}^{\prime}(u)_{n}^{n^{\prime \prime}}=\delta_{n^{\prime} n^{\prime \prime}}
$$

$a$ The standard vertex-face correspondence, as it is described in Ref. 22 , relates the SOS model in the regime $I I I$ to the eight-vertex model in the disordered region. But we can relate the disordered and ferroelectric region by use of the Baxter 


$$
\begin{aligned}
& t_{\varepsilon}\left(u_{0}-u\right)_{n}^{n^{\prime}}=\frac{n^{\prime}}{u_{0}-Y_{u}^{Y--}}
\end{aligned}
$$

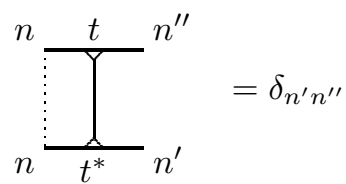

$$
\begin{aligned}
& t_{\varepsilon}^{*}\left(u_{0}-u\right)_{n}^{n^{\prime}}=u_{0} \frac{\mid}{n} \frac{\varepsilon}{y_{u}^{\dot{1}}} n^{\prime}
\end{aligned}
$$



(a)

(b)

Fig. 3. Intertwining vectors: $(a)$ graphic notations; $(b)$ definition of the conjugate and 'primed' vectors.

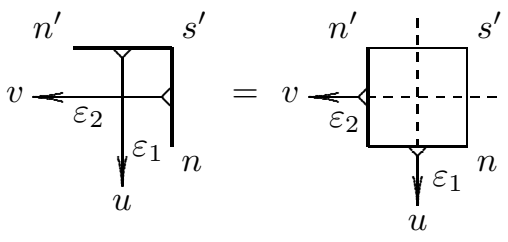

(a)

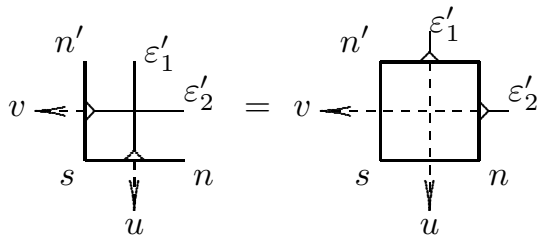

$(b)$

Fig. 4. Vertex-face correspondence: $(a)$ by usual intertwining vectors; $(b)$ by conjugate intertwining vectors.

Explicitly,

$$
\begin{aligned}
t_{\varepsilon}^{*}(u)_{n}^{n^{\prime}} & =(-)^{n-m+1} \frac{n^{\prime}-n}{C[n][u]} t_{-\varepsilon}(u-1)_{n}^{n^{\prime}}, \quad C=2 \theta_{2}(0) \theta_{3}(0)[r / 2]^{-2}, \\
t_{\varepsilon}^{\prime}(u)_{n}^{n^{\prime}} & =\frac{[u]}{[u-1]} \frac{\left[n^{\prime}\right]}{[n]} t_{\varepsilon}(u-2)_{n}^{n^{\prime}} .
\end{aligned}
$$

The conjugate intertwining vectors enter into another form of the vertex-face correspondence (Fig. 4b):

$$
\sum_{\varepsilon_{1} \varepsilon_{2}} t_{\varepsilon_{2}}^{*}\left(u_{0}-v\right)_{n^{\prime}}^{s} t_{\varepsilon_{1}}^{*}\left(u_{0}-u\right)_{s}^{n} R(u-v)_{\varepsilon_{1} \varepsilon_{2}}^{\varepsilon_{1}^{\prime} \varepsilon_{2}^{\prime}}=\sum_{s^{\prime} \in \mathbf{Z}} W\left[\begin{array}{cc}
n^{\prime} & s^{\prime} \\
s & n
\end{array} \mid u-v\right] t_{\varepsilon_{1}^{\prime}}^{*}\left(u_{0}-u\right)_{n^{\prime}}^{s^{\prime}} t_{\varepsilon_{2}^{\prime}}^{*}\left(u_{0}-v\right)_{s^{\prime}}^{n}
$$

\section{Vertex Operator Approach}

In the vertex operator approach (see Ref. 11 and references therein) the computation of correlation functions is reduced to the algebraic problem of finding the traces of some operators. Namely, the lattice is partitioned into several parts as it is shown in Fig. 5. Then the partition functions of the parts with fixed variables

duality transformation ${ }^{3}$

$$
\begin{aligned}
& a(u)=\frac{1}{2}\left(a_{D}(u)+b_{D}(u)+c_{D}(u)+d_{D}(u)\right), \\
& b(u)=\frac{1}{2}\left(-a_{D}(u)-b_{D}(u)+c_{D}(u)+c_{D}(u)\right), \\
& c(u)=\frac{1}{2}\left(a_{D}(u)-b_{D}(u)+c_{D}(u)-d_{D}(u)\right), \\
& d(u)=\frac{1}{2}\left(-a_{D}(u)+b_{D}(u)+c_{D}(u)-d_{D}(u)\right),
\end{aligned}
$$

where $a_{D}, b_{D}, c_{D}, d_{D}$ mean the weights in the disordered phase. This duality for correlation functions ${ }^{24}$ gives

$$
t_{\varepsilon}(u)_{n}^{n^{\prime}}=\frac{\varepsilon^{n-m}}{\sqrt{2}}\left(t_{+}^{D}(u)_{n}^{n^{\prime}}+\varepsilon t_{-}^{D}(u)_{n}^{n^{\prime}}\right)
$$




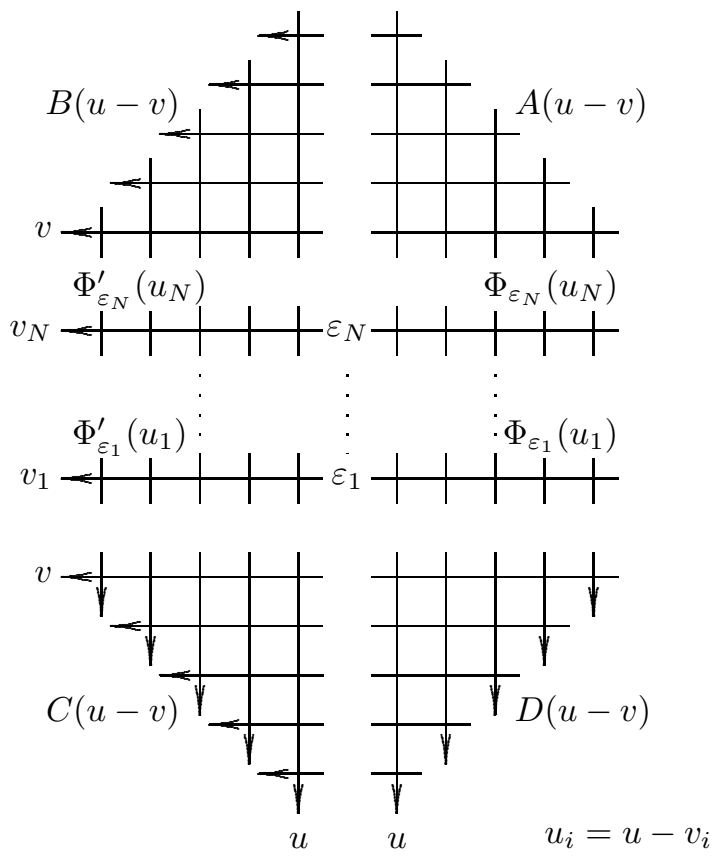

$(a)$

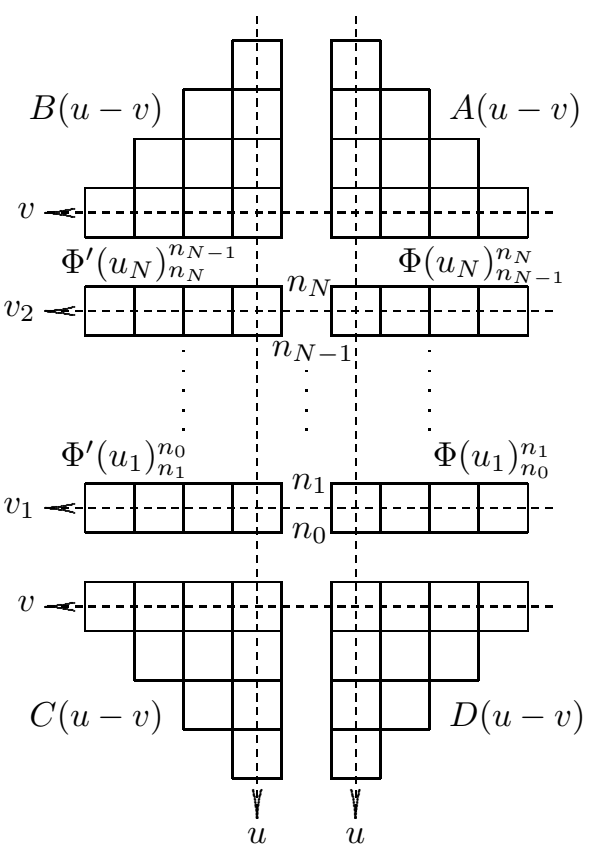

(b)

Fig. 5. Partition of the lattice into the corner transfer matrices $A, B, C, D$ and vertex operators $\Phi$. The lattice is only cut to stress the mode of partition, it must be considered as an integral thing. Action of the operators is anti-clockwise. (a) Eight-vertex model. (b) SOS model.

at their boundaries are treated as matrix elements of some operators (corner transfer matrices ${ }^{3}$ and vertex operators $^{9}$ ) acting in the spaces associated with a half-line.

\subsection{Eight-Vertex Model}

Let us begin with the eight-vertex model in the antiferroelectric region. ${ }^{8}$ Consider the subspace $\mathcal{H}_{i}$ in $\mathbf{C}^{2} \otimes \mathbf{C}^{2} \otimes \cdots$, spanned on the vectors $\left|\varepsilon_{1}\right\rangle \otimes\left|\varepsilon_{2}\right\rangle \otimes \cdots$ such that $\varepsilon_{k}$ stabilizes to $(-)^{k+i}$ for large $k$. The operators associated to the parts of the lattice, shown in Fig. 5a, are

$$
\begin{gathered}
A^{(1-i, i)}(u), C^{(1-i, i)}(u): \mathcal{H}_{i} \rightarrow \mathcal{H}_{1-i}, \quad B^{(i)}(u), D^{(i)}(u): \mathcal{H}_{i} \rightarrow \mathcal{H}_{i}, \\
\Phi_{\varepsilon}^{(1-i, i)}(u), \Phi_{\varepsilon}^{(1-i, i) \prime}(u): \mathcal{H}_{i} \rightarrow \mathcal{H}_{1-i} .
\end{gathered}
$$

The product of four corner transfer matrices in the infinite lattice is an operator independent of $u$ :

$$
\rho^{(i)} \equiv D^{(i)}(u) C^{(i, 1-i)}(u) B^{(1-i)}(u) A^{(1-i, i)}(u)=\operatorname{const} x^{4 H^{(i)}},
$$

where const is a $c$-number and $H^{(i)}$ is so called corner Hamiltonian.

The spectrum of the operator $H^{(i)}$ is equidistant. More precisely, it is determined by the generating function

$$
\chi^{(i)}(q) \equiv \operatorname{Tr}_{\mathcal{H}_{i}} q^{H^{(i)}}=\left(q^{1 / 2} ; q\right)_{\infty}^{-1} .
$$

The vertex operators $\Phi_{\varepsilon}^{(1-i, i)}(u)$ satisfy the following relations which are consequences of the YangBaxter equation, unitarity and crossing symmetry:

$$
\begin{gathered}
\Phi_{\varepsilon_{1}}^{(i, 1-i)}\left(u_{1}\right) \Phi_{\varepsilon_{2}}^{(1-i, i)}\left(u_{2}\right)=\sum_{\varepsilon_{1}^{\prime} \varepsilon_{2}^{\prime}} R\left(u_{1}-u_{2}\right)_{\varepsilon_{1} \varepsilon_{2}}^{\varepsilon_{1}^{\prime} \varepsilon_{2}^{\prime}} \Phi_{\varepsilon_{2}^{\prime}}^{(i, 1-i)}\left(u_{2}\right) \Phi_{\varepsilon_{1}^{\prime}}^{(1-i, i)}\left(u_{1}\right), \\
\Phi_{\varepsilon}^{(1-i, i)}(u) \rho^{(i)}=\rho^{(1-i)} \Phi_{\varepsilon}^{(1-i, i)}(u-2), \\
\sum_{\varepsilon} \Phi_{-\varepsilon}^{(i, 1-i)}(u-1) \Phi_{\varepsilon}^{(1-i, i)}(u)=1 .
\end{gathered}
$$


The conjugate vertex operator $\Phi_{\varepsilon}^{(i, 1-i) *}(u)$ defined as

$$
\Phi_{\varepsilon}^{(1-i, i) \prime}(u) B^{(i)}(u) A^{(i, 1-i)}(u)=B^{(1-i)}(u) A^{(1-i, i)}(u) \Phi_{\varepsilon}^{(i, 1-i) *}(u), \quad \sum_{\varepsilon} \Phi_{\varepsilon}^{(i, 1-i) *}(u) \Phi_{\varepsilon}^{(1-i, i)}(u)=1
$$

is given by

$$
\Phi_{\varepsilon}^{(i, 1-i) *}(u)=\Phi_{-\varepsilon}^{(i, 1-i)}(u-1)
$$

The probability of the configuration of polarizations on an inhomogeneous lattice as it is shown in Fig. 5a is given by

$$
\begin{gathered}
P_{\varepsilon_{1} \ldots \varepsilon_{N}}^{(i)}=\frac{1}{\chi^{(i)}\left(x^{4}\right)} \operatorname{Tr}_{\mathcal{H}_{i}}\left(\Phi_{\varepsilon_{1}}^{(i, 1-i) *}\left(u_{1}\right) \ldots \Phi_{\varepsilon_{N}}^{\left(1-i^{\prime}, i^{\prime}\right) *}\left(u_{N}\right) \Phi_{\varepsilon_{N}}^{\left(i^{\prime}, 1-i^{\prime}\right)}\left(u_{N}\right) \ldots \Phi_{\varepsilon_{1}}^{(1-i, i)}\left(u_{1}\right) x^{4 H^{(i)}}\right), \\
u_{k}=u-v_{k}, \quad(-)^{i^{\prime}}=(-)^{N+i} .
\end{gathered}
$$

Consider more general functions

$$
F_{\varepsilon_{1} \ldots \varepsilon_{N}}^{(i)}\left(u_{1}, \ldots, u_{N}\right)=\frac{1}{\chi^{(i)}\left(x^{4}\right)} \operatorname{Tr}_{\mathcal{H}_{i}}\left(\Phi_{\varepsilon_{N}}^{(i, 1-i)}\left(u_{N}\right) \ldots \Phi_{\varepsilon_{1}}^{(1-i, i)}\left(u_{1}\right) x^{4 H^{(i)}}\right)
$$

for even $N$. They satisfy the equations ${ }^{8}$

$$
\begin{gathered}
F_{\varepsilon_{1} \ldots \varepsilon_{N}}^{(i)}\left(\ldots, u_{j}+\frac{2 \pi \mathrm{i}}{\epsilon}, \ldots\right)=F_{\varepsilon_{1} \ldots \varepsilon_{N}}^{(i)}\left(\ldots, u_{j}, \ldots\right), \\
F_{\varepsilon_{1} \ldots \varepsilon_{N}}^{(i)}\left(u_{1}+a, \ldots, u_{N}+a\right)=F_{\varepsilon_{1} \ldots \varepsilon_{N}}^{(i)}\left(u_{1}, \ldots, u_{N}\right) \\
\sum_{\varepsilon_{j}^{\prime} \varepsilon_{j+1}^{\prime}} R\left(u_{j}-u_{j+1}\right)_{\varepsilon_{j} \varepsilon_{j+1} \varepsilon_{j+1}^{\prime}}^{F_{\ldots}} F_{\ldots \varepsilon_{j}^{\prime} \varepsilon_{j+1}^{\prime} \ldots}^{(i)}\left(\ldots, u_{j}, u_{j+1}, \ldots\right) \\
=F_{\ldots \varepsilon_{j+1} \varepsilon_{j} \ldots}^{(i)}\left(\ldots, u_{j+1}, u_{j}, \ldots\right), \\
F_{\varepsilon_{1} \varepsilon_{2} \ldots \varepsilon_{N}}^{(i)}\left(u_{1}+2, u_{2}, \ldots, u_{N}\right)=F_{\varepsilon_{2} \ldots \varepsilon_{N} \varepsilon_{1}}^{(1-i)}\left(u_{2}, \ldots, u_{N}, u_{1}\right), \\
\sum_{\varepsilon} F_{\varepsilon_{1} \ldots \varepsilon_{N}, \varepsilon,-\varepsilon}^{(i)}\left(u_{1}, \ldots, u_{N}, u, u-1\right)=F_{\varepsilon_{1} \ldots \varepsilon_{N}}^{(i)}\left(u_{1}, \ldots, u_{N}\right), \quad \sum_{\varepsilon} F_{-\varepsilon, \varepsilon}^{(i)}(u, u-1)=1 .
\end{gathered}
$$

These equations follow from Eqs. (3.4-3.5) and can be considered as defining equations for correlation functions.

\subsection{SOS model}

For the SOS model the construction repeats that for the eight-vertex model in general outline (see Fig. 5b). The corner transfer matrices are labelled by two integers $m$ and $n$. The variable $n$ denotes the height at the corner. The number $m$ describes the condition at the infinity. Namely, for the space $\mathcal{H}_{m, n}$ with $0<m<r-1$ is the subspace of $\mathbf{C}^{\infty} \otimes \mathbf{C}^{\infty} \otimes \cdots$ spanned of vectors $\left|n_{0}\right\rangle \otimes\left|n_{1}\right\rangle \otimes\left|n_{2}\right\rangle \otimes \cdots$, such that $n_{0}=n$, each pair $\left(n_{k}, n_{k+1}\right)$ is an admissible pair of heights, and for $k$ large enough $n_{2 k}=m$ and $n_{2 k+1}=m+1$ if $n-m$ is even, $n_{2 k}=m+1$ and $n_{2 k+1}=m$ if $n-m$ is odd. The situation of general $m$ is more complicated, but we need not discuss it for our purposes. For simplicity we restrict the discussion to $r>2$, but our final construction is valid for any $r>1$. Parts of the lattice shown in Fig. 5b define again operators:

$$
\begin{gathered}
A_{m, n}(u), B_{m, n}(u), C_{m, n}(u), D_{m, n}(u): \mathcal{H}_{m, n} \rightarrow \mathcal{H}_{m, n} \\
\Phi(u)_{m, n}^{m, n^{\prime}}, \Phi^{\prime}(u)_{m, n}^{m, n^{\prime}}: \mathcal{H}_{m, n} \rightarrow \mathcal{H}_{m, n^{\prime}}
\end{gathered}
$$

The product of the corner transfer matrices is again a $u$-independent operator:

$$
\rho_{m, n} \equiv D_{m, n}(u) C_{m, n}(u) B_{m, n}(u) A_{m, n}(u)=\operatorname{const}[n] x^{4 H_{m, n}} .
$$


The operator $H_{m, n}$ also possesses an equidistant spectrum. We assume that the respective generating function is

$$
\chi_{m, n}(q) \equiv \operatorname{Tr}_{\mathcal{H}_{m, n}} q^{H_{m, n}}=q^{(r m-(r-1) n)^{2} / 4 r(r-1)}(q ; q)_{\infty}^{-1} .
$$

The vertex operators $\Phi(u)_{n}^{n^{\prime}}$ (we usually omit indices $m$ in operators from here on) obey the relations:

$$
\begin{gathered}
\Phi\left(u_{1}\right)_{s}^{n^{\prime}} \Phi\left(u_{2}\right)_{n}^{s}=\sum_{s^{\prime}} W\left[\begin{array}{cc}
n^{\prime} & s^{\prime} \\
s & n
\end{array} \mid u_{1}-u_{2}\right] \Phi\left(u_{2}\right)_{s^{\prime}}^{n^{\prime}} \Phi\left(u_{1}\right)_{n}^{s^{\prime}} \\
\Phi(u)_{n}^{n^{\prime}} \rho_{n}=\rho_{n^{\prime}} \Phi(u-2)_{n}^{n^{\prime}} \\
\sum_{n^{\prime}}\left(n^{\prime}-n\right)\left[n^{\prime}\right] \Phi(u-1)_{n^{\prime}}^{n} \Phi(u)_{n}^{n^{\prime}}=(-1)^{n-m}
\end{gathered}
$$

The conjugate operator defined as

$$
\Phi^{\prime}(u)_{n}^{n^{\prime}} B_{n}(u) A_{n}(u)=B_{n^{\prime}}(u) A_{n^{\prime}}(u) \Phi^{*}(u)_{n}^{n^{\prime}}, \quad \sum_{n^{\prime}} \Phi^{*}(u)_{n^{\prime}}^{n} \Phi(u)_{n}^{n^{\prime}}=1
$$

is given by

$$
\Phi^{*}(u)_{n}^{n^{\prime}}=(-)^{n-m}\left(n^{\prime}-n\right)[n] \Phi(u-1)_{n}^{n^{\prime}}
$$

\section{Vertex-Face Correspondence and Correlation Functions}

Now we are ready to explore the vertex-face correspondence for the vertex operator construction.

Let us enclose a piece of lattice of the eight-vertex model with several fixed polarizations with the intertwining vectors from the upper and right sides and with the conjugate intertwining vectors from the lower and left sides (Fig. 6a). We can shrink such loop using the vertex-face correspondence and the definition of the conjugate intertwining vector (2.10) (Fig. 6b). But the tail in the right lower part of Fig. 6b cannot be cancelled, because the heights $n$ and $n^{\prime}$ designated at the figure are generically not equal.

Now let us establish correspondence between ground states (or conditions at infinity) for the SOS and eight-vertex models. Let $m$ in the definition of the intertwining vectors (2.8) coincide with $m$ characterizing the condition at the infinity. To do it, consider the 'low temperature limit' $x \rightarrow 0(\epsilon \rightarrow \infty)$. For simplicity we consider the case $0<m<r-1$.

It can be checked (see Appendix A) that the relevant values of $u$ in $t_{\varepsilon}(u)_{n}^{n^{\prime}}$ and $t_{\varepsilon}^{*}(u)_{n}^{n^{\prime}}$ for the free field representation are

$$
-2<\operatorname{Re} u<0
$$

In the limit $x \rightarrow 0$ we have for the intertwining vectors

$$
\begin{aligned}
& t_{+}(u)_{n}^{n^{\prime}} \simeq \sqrt{\frac{\epsilon r}{\pi}} \mathrm{e}^{-\frac{\epsilon}{2 r}\left(\left(n^{\prime}-n\right) u+n^{\prime}\right)^{2}}, \\
& t_{-}(u)_{n}^{n^{\prime}} \simeq(-)^{n-m+1} \sqrt{\frac{\epsilon r}{\pi}} \mathrm{e}^{-\frac{\epsilon}{2 r}\left(\left(n^{\prime}-n\right) u+n^{\prime}\right)^{2}-\epsilon r+\epsilon\left(\left(n^{\prime}-n\right) u+n^{\prime}\right)}, \\
& t_{+}^{*}(u)_{n}^{n^{\prime}} \simeq\left(n-n^{\prime}\right) \sqrt{\frac{\pi}{\epsilon r}} \mathrm{e}^{\frac{\epsilon}{2 r}\left(\left(n-n^{\prime}\right) u+n\right)^{2}+\left(1+n^{\prime}-n\right) \epsilon u}, \\
& t_{-}^{*}(u)_{n}^{n^{\prime}} \simeq(-)^{n-m+1}\left(n^{\prime}-n\right) \sqrt{\frac{\pi}{\epsilon r}} \mathrm{e}^{\frac{\epsilon}{2 r}\left(\left(n-n^{\prime}\right) u+n\right)^{2}+\epsilon r-\epsilon(n-u)}, \quad n, n^{\prime}>0 .
\end{aligned}
$$

Consider now the quantities (Fig. 7a)

$$
a_{\varepsilon}^{(1-i, i)}=t_{\varepsilon}^{*}(u)_{m+1-i}^{m+i} t_{\varepsilon}(u)_{m+i}^{m+1-i}
$$

It is easy to check that

$$
\left|a_{+}^{(1,0)}\right| \simeq 1, \quad\left|a_{-}^{(1,0)}\right| \simeq \mathrm{e}^{2 \epsilon \operatorname{Re} u}, \quad\left|a_{+}^{(0,1)}\right| \simeq \mathrm{e}^{2 \epsilon \operatorname{Re} u}, \quad\left|a_{-}^{(0,1)}\right| \simeq 1 .
$$




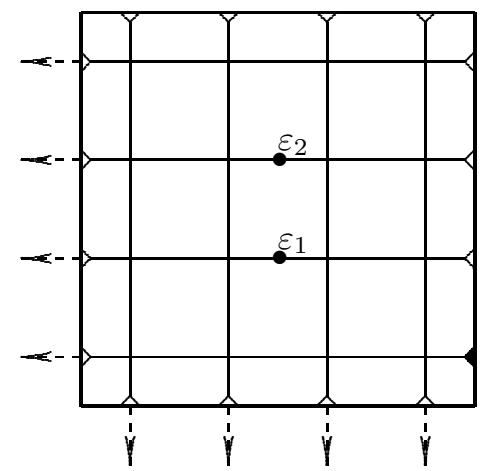

(a)

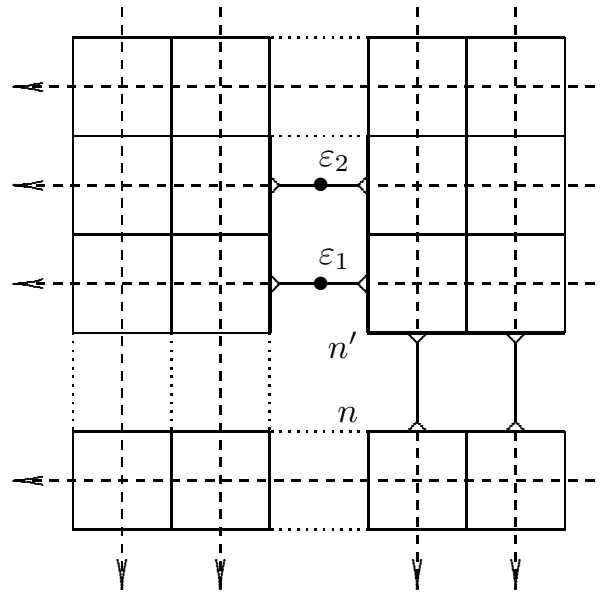

(b)

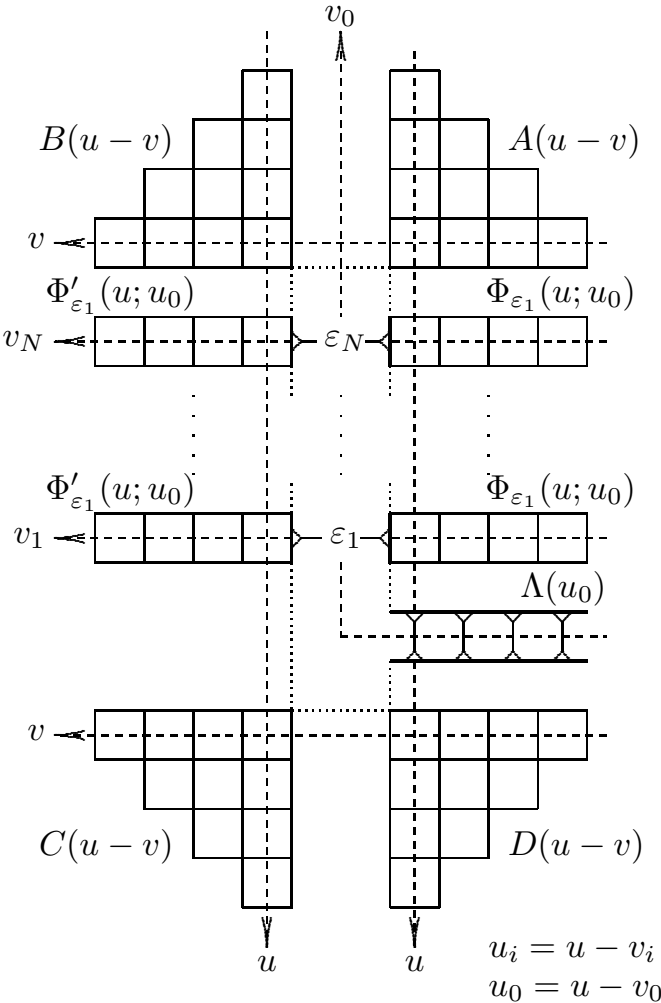

(c)

Fig. 6. Contraction of the loop of intertwining vectors in the lattice with insertions. (a) Initial loop. The $t^{\prime}$ vector in the lower write side is used to simplify the next figure. (b) 'Maximal' contraction. Dotted lines connect points with coincident heights. Summation in $n$ and $n^{\prime}$ is implied, so they do not necessarily coincide. (c) Partition of the lattice after such contraction.

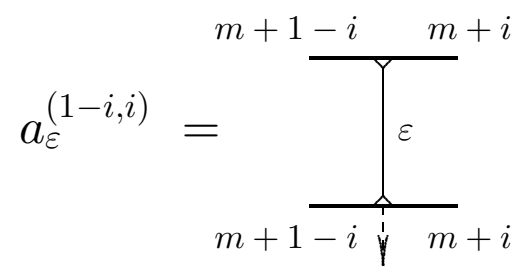

(a)

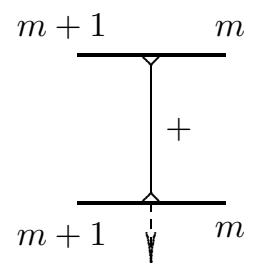

(b)

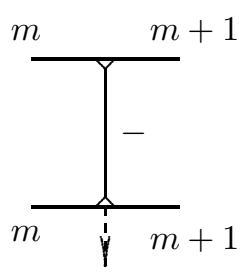

Fig. 7. Vertex-face correspondence of ground states: $(a)$ basic weights; $(b)$ configurations of maximal weight at low temperatures $(x \rightarrow 0)$ for $m>0$.

We see that

$$
\left|a_{+}^{(1,0)}\right| \gg\left|a_{-}^{(1,0)}\right|, \quad\left|a_{-}^{(0,1)}\right| \gg\left|a_{+}^{(0,1)}\right| .
$$

The configurations shown in Fig. 7b give the leading contribution into the partition function. The other two configurations are vanishing in the low-temperature limit. It means that a definite ground state configuration of the SOS model imposes a definite ground state configuration of the eight-vertex model. Hence the SOS condition at the infinity $m$, such that $n-m \in 2 \mathbf{Z}+i$, imposes the condition at the infinity $i$ for the eight-vertex model.

So we can partition the lattice as it is shown in Fig. 6c. According to this partition we introduce the following objects: the corner transfer matrices of the SOS model, the vertex operators, and the 'tail operator'. 
Let us begin with the corner transfer matrices of the SOS model

$$
\rho^{\prime(i)}=\bigoplus_{n \in 2 \mathbf{Z}+m+i} D_{m, n}(u) C_{m, n}(u) B_{m, n}(u) A_{m, n}(u)=\mathrm{const} \bigoplus_{n \in 2 \mathbf{Z}+m+i}[n] x^{4 H_{n}} .
$$

We assume that one have to keep fixed an arbitrary nonzero value of $m$ to calculate correlation functions. This conjecture seems to be physically reasonable, because such condition is compatible with the vertex-face correspondence: we may take respective values of heights at the boundary of Fig. 6a, not disturbing the shrinking procedure. Besides, the correlation function in the eight-vertex model must be sensitive to the eight-vertex boundary condition labeled by $i \equiv n-m \quad(\bmod 2)$, but insensitive to details of arrangement of the facilities that create these conditions, like a system of intertwining vectors and the SOS lattice somewhere at infinity.

The vertex operators are given by

$$
\begin{aligned}
\Phi_{\varepsilon}^{(1-i, i)}\left(u ; u_{0}\right) & =f\left(u-u_{0}\right) \bigoplus_{\substack{n, n^{\prime} \\
n \in 2 \mathbf{Z}+m+i}} t_{\varepsilon}\left(u-u_{0}\right)_{n}^{n^{\prime}} \Phi(u)_{n}^{n^{\prime}}, \\
\Phi_{\varepsilon}^{(1-i, i) *}\left(u ; u_{0}\right) & =f^{-1}\left(u-u_{0}\right) \bigoplus_{\substack{n, n^{\prime} \\
n \in 2 \mathbf{Z}+m+i}} t_{\varepsilon}^{*}\left(u-u_{0}\right)_{n}^{n^{\prime}} \Phi^{*}(u)_{n}^{n^{\prime}}=\Phi_{-\varepsilon}^{(1-i, i)}(u-1)
\end{aligned}
$$

with the function

$$
\begin{aligned}
f(u) & =\frac{1}{\sqrt{C}} x^{-u^{2} / 2 r+(r-1) u / 2 r+1 / 4} f_{1}\left(x^{2 u}\right) \\
f_{1}(z) & =\frac{1}{\sqrt{\left(x^{2 r} ; x^{2 r}\right)_{\infty}}} \frac{\left(x^{4} z ; x^{4}, x^{2 r}\right)_{\infty}\left(x^{2+2 r} z^{-1} ; x^{4}, x^{2 r}\right)_{\infty}}{\left(x^{2} z ; x^{4}, x^{2 r}\right)_{\infty}\left(x^{2 r} z^{-1} ; x^{4}, x^{2 r}\right)_{\infty}}
\end{aligned}
$$

satisfying the equations

$$
C[u] f(u) f(u-1)=1, \quad \frac{f(u-2)}{f(u)}=\frac{[u]}{[u-1]} .
$$

These vertex operators satisfy Eqs. (3.3) and (3.5) and we want to identify them with the operators $\Phi_{\varepsilon}^{(1-i, i)}(u)$. But they contain a new free parameter $u_{0}$, whereas the final formulas for correlation functions like (3.8) and (3.9) must be $u_{0}$ independent. So let us look at the situation more carefully. Any $u_{0}$-dependent operator $O^{\left(i^{\prime}, i\right)}\left(u_{0}\right): \mathcal{H}_{m}^{(i)} \rightarrow \mathcal{H}_{m}^{\left(i^{\prime}\right)}$ with $\mathcal{H}_{m}^{(i)}=\bigoplus_{n \in 2 \mathbf{Z}+m+i} \mathcal{H}_{m, n}$, which is defined in the SOS model, is related with the respective $u_{0}$-independent operator $O^{\left(i^{\prime}, i\right)}: \mathcal{H}_{i} \rightarrow \mathcal{H}_{i^{\prime}}$, which is defined in the eight-vertex model, by the intertwining property

$$
O^{\left(i^{\prime}, i\right)}\left(u_{0}\right) U_{m}^{(i)}\left(u_{0}\right)=U_{m}^{\left(i^{\prime}\right)}\left(u_{0}\right) O^{\left(i^{\prime}, i\right)},
$$

where

$$
U_{m}^{(i)}\left(u_{0}\right)_{\varepsilon_{1} \varepsilon_{2} \ldots}^{n_{1} n_{2} \ldots}=\bigoplus_{n} \hat{t}_{\varepsilon_{1}}\left(u_{0}-u\right)_{n_{1}}^{n} \hat{t}_{\varepsilon_{2}}\left(u_{0}-u\right)_{n_{2}}^{n_{1}} \ldots \quad: \quad \mathcal{H}_{m}^{(i)} \rightarrow \mathcal{H}_{i}
$$

Here $u$ is the spectral parameter at the vertical arrows in Fig. 6 c, sequences $\varepsilon_{1}, \varepsilon_{2}, \ldots$ and $n, n_{1}, n_{2}, \ldots$ stabilize to the respective ground state sequences, and each intertwining vector is divided by its value on the ground state configuration, which is denoted by hats. So the traces in both cases must coincide if the $u_{0}$-dependent operators are properly defined.

The 'tail operator' $\Lambda(u)=\bigoplus_{n, n^{\prime}} \Lambda(u)_{n}^{n^{\prime}}$ is

$$
\Lambda(u)_{n}^{n^{\prime} n_{1}^{\prime} n_{2}^{\prime} \ldots} n_{1} n_{2} \ldots=L\left[\begin{array}{cc}
n^{\prime} & n_{1}^{\prime} \\
n & n_{1}
\end{array} \mid u\right] L\left[\begin{array}{cc}
n_{1}^{\prime} & n_{2}^{\prime} \\
n_{1} & n_{2}
\end{array} \mid u\right] \ldots
$$

with

$$
L\left[\begin{array}{cc}
n_{4} & n_{3} \\
n_{1} & n_{2}
\end{array} \mid u\right]=\sum_{\varepsilon} t_{\varepsilon}^{*}(-u)_{n_{1}}^{n_{2}} t_{\varepsilon}(-u)_{n_{3}}^{n_{4}}
$$



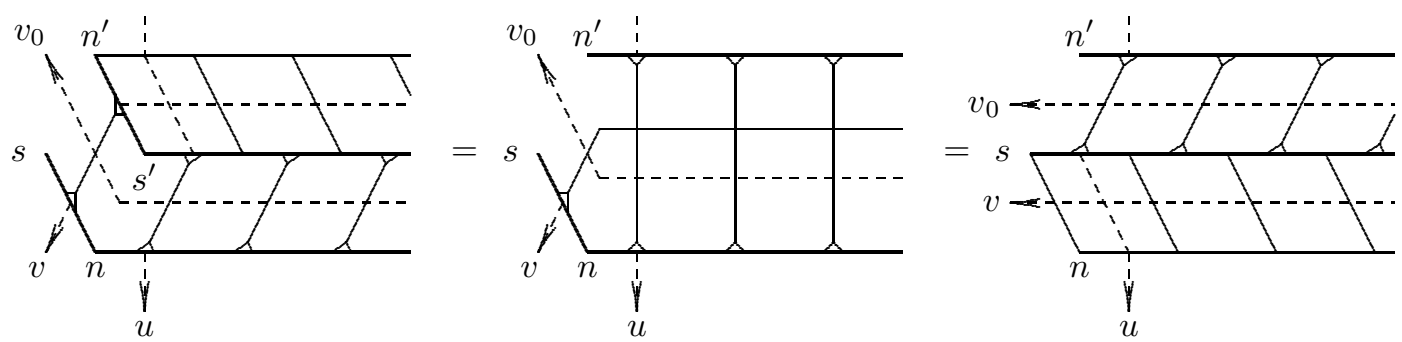

Fig. 8. Commutation of $\Phi(u-v)_{n}^{n^{\prime}}$ with $\Lambda\left(u_{0}-u\right)$. Summation in $s^{\prime}$ is implied.

In fact, the r. h. s. of (4.7) is a finite product because all sequences $n_{1}, n_{2}, \ldots, n_{k}, \ldots$ stabilize to $(-)^{k+i}$ by definition. Note also that we only need the elements with $n^{\prime}-n \in 2 \mathbf{Z}$ [or $n_{1}-n_{4} \in 2 \mathbf{Z}$ in Eq. (4.8)]. This is because only even number of vertex operators enter into the correlation functions (see Fig. 6c). So we shall imply it from here on.

The functions $L$ can be easily calculated explicitly:

$$
\begin{aligned}
L\left[\begin{array}{cc}
n^{\prime} & n^{\prime} \pm 1 \\
n & n \pm 1
\end{array} \mid u\right] & =\frac{\left[u \mp \frac{1}{2}\left(n-n^{\prime}\right)\right]\left[\frac{1}{2}\left(n+n^{\prime}\right)\right]}{[u][n]}, \quad \text { for } \quad n^{\prime}-n \in 2 \mathbf{Z} . \\
L\left[\begin{array}{cc}
n^{\prime} & n^{\prime} \mp 1 \\
n & n \pm 1
\end{array} \mid u\right] & =\frac{\left[u \mp \frac{1}{2}\left(n+n^{\prime}\right)\right]\left[\frac{1}{2}\left(n-n^{\prime}\right)\right]}{[u][n]}
\end{aligned}
$$

By definition

$$
L\left[\begin{array}{cc|c}
n & n^{\prime \prime} \\
n & n^{\prime} & u
\end{array}\right]=\delta_{n^{\prime} n^{\prime \prime}}
$$

and hence

$$
\Lambda(u)_{n}^{n}=1
$$

The counterpart of the operator $\rho^{(i)}$ is

$$
\rho^{(i)}\left(u_{0}\right)=\mathrm{const} \bigoplus_{\substack{n^{\prime}, n \\ n \in 2 \mathbf{Z}+m+i}} \Lambda\left(u_{0}\right)_{n}^{n^{\prime}}[n] x^{4 H_{m, n}}
$$

To prove the defining equations (3.10) we need only prove the properties (3.4-3.5) of the vertex operators. The commutation relation (3.3) and the normalization condition (3.5) are proven directly. ${ }^{23 b}$ The most subtle point is the proof of Eq. (3.4) for $\Phi^{(1-i, i)}\left(u, u_{0}\right)$ and $\rho^{(i)}\left(u_{0}\right)$. To do it we need the commutation relation between $\Phi_{\varepsilon}\left(u ; u_{0}\right)$ and $\Lambda\left(u_{0}\right)$. As it shown in Fig. 8, we have the following relation

$$
\Lambda\left(u_{0}\right)_{s}^{n^{\prime}} \Phi(u)_{n}^{s}=\sum_{s^{\prime}} L\left[\begin{array}{cc}
n^{\prime} & s^{\prime} \\
s & n
\end{array} \mid u_{0}-u\right] \Phi(u)_{s^{\prime}}^{n^{\prime}} \Lambda\left(u_{0}\right)_{n}^{s^{\prime}} .
$$

Multiplying it by $f\left(u-u_{0}-2\right) t_{\varepsilon}^{\prime}\left(u-u_{0}\right)_{n}^{s}$, taking sum in $s$, and using Eqs. (2.10), (2.11) we have

$$
\Phi_{\varepsilon}^{(1-i, i)}\left(u ; u_{0}\right) \Lambda\left(u_{0}\right)=\Lambda\left(u_{0}\right) f\left(u-u_{0}-2\right) \bigoplus_{n, n^{\prime}} \frac{\left[n^{\prime}\right]}{[n]} t_{\varepsilon}\left(u-u_{0}-2\right)_{n}^{n^{\prime}} \Phi(u)_{n}^{n^{\prime}} .
$$

We see that commutation with $\Lambda$ shifts $u$ by -2 in each function except $\Phi(u)_{n}^{n^{\prime}}$ and adds the factor $\left[n^{\prime}\right] /[n]$. Now pulling $\Phi(u)_{n}^{n^{\prime}}$ through $[n] x^{4 H_{n}}$ we obtain (3.4).

It is necessary also to ensure that the spectrum of $\rho^{(i)}\left(u_{0}\right)$ from (4.12) coincides with the spectrum of $\rho^{(i)}$ from (3.1). To do it let us calculate the quantity

$$
\chi_{m}^{\prime(i)}\left(x^{4}\right)=\sum_{n \in 2 \mathbf{Z}+m+i} \operatorname{Tr}_{\mathcal{H}_{m, n}}\left(\Lambda\left(u_{0}\right)_{n}^{n}[n] x^{4 H_{m, n}}\right)=\sum_{n \in 2 \mathbf{Z}+m+i}[n] \chi_{m, n}\left(x^{4}\right) .
$$

${ }^{b}$ As well as we know, M. Jimbo was the first to make this observation. 
In the Appendix B it is shown that

$$
\chi_{m}^{\prime(i)}\left(x^{4}\right)=[m]^{\prime} \chi^{(i)}\left(x^{4}\right)
$$

with

$$
[u]^{\prime}=x^{\frac{u^{2}}{r-1}-u} \Theta_{x^{2(r-1)}}\left(x^{2 u}\right)=\sqrt{\frac{\pi}{\epsilon(r-1)}} \mathrm{e}^{\frac{1}{4} \epsilon(r-1)} \theta_{1}\left(\frac{u}{r-1} ; \frac{\mathrm{i} \pi}{\epsilon(r-1)}\right) .
$$

We expect that traces of operators (4.4) and (4.12) are proportional to $[\mathrm{m}]^{\prime}$ so that this factor cancels in the probabilities. This expectation is realized at least in the case of two vertex operators in the trace (see Appendix D). In other words, we expect that

$$
\Lambda\left(u_{0}\right) \rho_{m}=\text { const }[m]^{\prime} x^{4 H^{(i)}\left(u_{0}\right)}
$$

with $H^{(i)}\left(u_{0}\right)$ being an operator with the same spectrum as the eight-vertex corner Hamiltonian $H^{(i)}$ and $\rho_{m}=\bigoplus_{n} \rho_{m, n}$.

\section{Free Field Representation for the Eight-Vertex Model}

\subsection{Free Field Representation for the SOS model ${ }^{16}$}

The most efficient way to calculate correlation functions in the SOS model is based on the free field representation. Consider a Heisenberg algebra of operators $a_{n}$ with nonzero integer $n$ and a pair of 'zero mode' operators $\mathcal{P}$ and $\mathcal{Q}$ with the commutation relations

$$
[\mathcal{P}, \mathcal{Q}]=-\mathrm{i}, \quad\left[a_{k}, a_{l}\right]=k \frac{[k]_{x}[(r-1) k]_{x}}{[2 k]_{x}[r k]_{x}} \delta_{k+l, 0} \quad \text { with } \quad[u]_{x}=\frac{x^{u}-x^{-u}}{x-x^{-1}}
$$

The normal ordering operation $: \ldots$ : places $\mathcal{P}$ to the right of $\mathcal{Q}$ and $a_{k}$ with positive $k$ to the right of $a_{-k}$. Now introduce the field

$$
\varphi(z)=-\sqrt{\frac{r-1}{2 r}}(\mathcal{Q}-\mathrm{i} \mathcal{P} \log z)-\sum_{k \neq 0} \frac{a_{k}}{\mathrm{i} k} z^{-k} .
$$

This field enters into the exponential operators

$$
V(u)=z^{(r-1) / 4 r}: \mathrm{e}^{\mathrm{i} \varphi(z)}:, \quad \bar{V}(u)=z^{(r-1) / r}: \mathrm{e}^{-\mathrm{i} \varphi\left(x^{-1} z\right)-\mathrm{i} \varphi(x z)}:,
$$

and Lukyanov's screening operator

$$
\begin{aligned}
X(u) & =\eta^{-1} \epsilon \int_{C_{u}} \frac{d v}{\mathrm{i} \pi} \bar{V}(v) \frac{\left[v-u+\frac{1}{2}-\sqrt{2 r(r-1)} \mathcal{P}\right]}{\left[v-u-\frac{1}{2}\right]} \\
\eta^{-1} & =\mathrm{i}[1] x^{\frac{r-1}{2 r}} \frac{\left(x^{2} ; x^{2 r}\right)_{\infty}}{\left(x^{2 r-2} ; x^{2 r}\right)_{\infty}} \frac{\left(x^{6} ; x^{4}, x^{2 r}\right)_{\infty}\left(x^{2 r+2} ; x^{4}, x^{2 r}\right)_{\infty}}{\left(x^{4} ; x^{4}, x^{2 r}\right)_{\infty}\left(x^{2 r+4} ; x^{4}, x^{2 r}\right)_{\infty}}
\end{aligned}
$$

The contour $C_{u}$ goes from $u$ to $u+\frac{\mathrm{i} \pi}{\epsilon}$.

Now the vertex operators are represented as follows:

$$
\begin{aligned}
& \Phi(u)_{n}^{n+1}=\frac{\mathrm{i}^{m-n}}{[n]} V(u), \\
& \Phi(u)_{n}^{n-1}=(-)^{m-n+1} \frac{\mathrm{i}^{m-n}}{[n]} V(u) X(u) .
\end{aligned}
$$

The corner Hamiltonian is given by

$$
H=\frac{\mathcal{P}^{2}}{2}+\sum_{k=1}^{\infty} \frac{[k]_{x}[r k]_{x}}{[2 k]_{x}[(r-1) k]_{x}} a_{-k} a_{k} .
$$


The operators (5.5) and (5.6) act in the direct sum of the Fock spaces $\mathcal{F}_{m, n}$ generated by the operators $a_{-k}$ with $k>0$ from the highest weight vectors $\left|P_{m, n}\right\rangle$ such that

$$
a_{k}\left|P_{m, n}\right\rangle=0 \quad(k>0), \quad \mathcal{P}\left|P_{m, n}\right\rangle=P_{m, n}\left|P_{m, n}\right\rangle, \quad P_{m, n}=m \sqrt{\frac{r}{2(r-1)}}-n \sqrt{\frac{r-1}{2 r}} .
$$

It is not clear if the spaces $\bigoplus_{n} \mathcal{H}_{m, n}$ and $\bigoplus_{n} \mathcal{F}_{m, n}$ coincide as representations of the vertex operator algebra. At least the numbers of states at each level (according to the $H$ grading) of both spaces $\mathcal{H}_{m, n}$ and $\mathcal{F}_{m, n}$ coincide. We only assume that traces over both spaces coincide.

Later we shall need the following fact on the SOS model. The weights (2.6) are invariant under the substitution $n_{k} \rightarrow-n_{k}$. So the model is invariant with respect to the change

$$
\begin{aligned}
n_{k} & \rightarrow-n_{k} \\
m & \rightarrow-m .
\end{aligned}
$$

The free field representation (5.5) is not invariant under this substitution. So there is another free field representation

$$
\begin{aligned}
& \Phi(u)_{n}^{n+1}=\frac{\mathrm{i}^{m-n}}{[n]} V(u) X(u), \\
& \Phi(u)_{n}^{n-1}=(-)^{m-n+1} \frac{\mathrm{i}^{m-n}}{[n]} V(u) .
\end{aligned}
$$

In this representation the space $\mathcal{H}_{m, n}$ is identified with $\mathcal{F}_{-m,-n}$. Of course, this representation gives the same local heights probabilities as the first representation.

\subsection{Free Field Representation for the Operator $\Lambda(u)$}

Now let us turn to the eight-vertex model. The representation of $\rho^{(i)}$ and $\Phi_{\varepsilon}^{(1-i, i)}\left(u ; u_{0}\right)$ completely reduces to the free field representation of the SOS model by use of Eqs. (4.3) and (4.4). The only unknown object is $\Lambda\left(u_{0}\right)$. To find its representation let us consider the limit $u_{0} \rightarrow u$ in the commutation relation (4.13). In this limit $L\left[\begin{array}{cc}n^{\prime} & s^{\prime} \\ s & n\end{array} \mid u\right] \rightarrow \infty$ and

$$
L\left[\begin{array}{cc}
n^{\prime} & n^{\prime}+1 \\
n \pm 1 & n
\end{array} \mid u\right] / L\left[\begin{array}{cc}
n^{\prime} & n^{\prime}-1 \\
n \pm 1 & n
\end{array} \mid u\right] \rightarrow 1, \quad u \rightarrow 0 .
$$

We have

$$
\Phi(u)_{n^{\prime}-1}^{n^{\prime}} \Lambda(u)_{n}^{n^{\prime}-1}=-\Phi(u)_{n^{\prime}+1}^{n^{\prime}} \Lambda(u)_{n}^{n^{\prime}+1}
$$

Substitution of (5.5) into (5.10) gives

$$
V(u) \Lambda(u)_{n}^{n^{\prime}-1}=(-)^{m-n^{\prime}} \frac{\left[n^{\prime}-1\right]}{\left[n^{\prime}+1\right]} V(u) X(u) \Lambda(u)_{n}^{n^{\prime}+1} .
$$

For $n^{\prime}<n$ and odd $n^{\prime}-n$ we have a solution with the initial condition (4.11):

$$
\Lambda(u)_{n}^{n-2 k}=(-)^{(m-n+1) k} \frac{[n-2 k]}{[n]} X^{k}(u) \quad \text { for } \quad k \geq 0 .
$$

In the Appendix $\mathrm{C}$ we prove that this solution satisfies the general commutation relation (4.13). So we may, consider Eq. (5.11) as the free field representation of $\Lambda$. Now we have to construct representatives for $\Lambda(u)_{n}^{n^{\prime}}$ with $n^{\prime}>n$. However, we know no such representatives in the representation (5.5). To solve this problem let us use the second free field representation of the SOS model (5.9), described at the end of Sec. 3. In this representation there is a natural representative

$$
\Lambda(u)_{n}^{n+2 k}=(-)^{(m-n+1) k} \frac{[n+2 k]}{[n]} X^{k}(u), \quad \text { for } \quad k \geq 0,
$$


acting from $\mathcal{F}_{-m,-n}$ to $\mathcal{F}_{-m,-n-2 k}$. So we must use the representation (5.5), (5.11) for the contributions with $n^{\prime} \leq n$, and the representation (5.9), (5.12) for those with $n^{\prime} \geq n$.

\subsection{Staggered Spontaneous Polarization}

Let us check our construction in the calculation of the staggered polarization in the antiferroelectric regime of the eight-vertex model. The staggered polarization is the average 'spin' $\langle\varepsilon\rangle^{(i)}$ in the state with the condition at the infinity $i$. According to (3.8) it is given by

$$
\langle\varepsilon\rangle^{(i)}=\sum_{\varepsilon} \varepsilon P_{\varepsilon}^{(i)}=\frac{1}{\operatorname{Tr} \rho^{(i)}\left(u_{0}\right)} \sum_{\varepsilon} \varepsilon \operatorname{Tr}\left(\Phi_{-\varepsilon}^{(i, 1-i)}\left(u-1, u_{0}\right) \Phi_{\varepsilon}^{(1-i, i)}\left(u, u_{0}\right) \rho^{(i)}\left(u_{0}\right)\right) .
$$

Substituting (4.4) we obtain

$$
\begin{aligned}
\langle\varepsilon\rangle^{(i)}= & \frac{(-1)^{i}}{[m]^{\prime}}\left(x^{2} ; x^{4}\right)_{\infty} \sum_{n_{2} \in \mathbf{2} \mathbf{Z}+m+i} \sum_{\substack{n_{1}=n_{2} \pm 1 \\
n_{0}=n_{1} \pm 1}}\left[n_{2}\right] K\left[\begin{array}{cc}
n_{1} & n_{0} \\
n_{1} & n_{2}
\end{array} \mid u-u_{0}\right] \\
& \times \operatorname{Tr}_{\mathcal{F}_{m, n_{2}}}\left(\Phi(u-1)_{n_{1}}^{n_{2}} \Phi(u)_{n_{0}}^{n_{1}} \Lambda\left(u_{0}\right)_{n_{2}}^{n_{0}} x^{4 H_{n}}\right)
\end{aligned}
$$

where

$$
(-)^{n_{3}-m} K\left[\begin{array}{ll}
n_{4} & n_{3} \\
n_{1} & n_{2}
\end{array} \mid u\right]=f(u) f(u-1) \sum_{\varepsilon} \varepsilon t_{-\varepsilon}(u-1)_{n_{1}}^{n_{2}} t_{\varepsilon}(u)_{n_{3}}^{n_{4}} .
$$

Applying the trick with the sign change of $m$ and $n$ s we obtain

$$
\begin{gathered}
\langle\varepsilon\rangle^{(i)}=\frac{(-1)^{i}}{[m]^{\prime}}\left(x^{2} ; x^{4}\right)_{\infty} \sum_{n \in 2 \mathbf{Z}+m+i}[n]\left(K_{n}^{+-}\left(T_{m, n}^{+-}-T_{-m, n}^{+-}\right)+K_{n}^{++}\left(T_{m, n}^{++}-T_{-m, n}^{++}\right)\right) \\
K_{n}^{+-}=K\left[\begin{array}{cc}
n-1 & n \\
n-1 & n
\end{array} \mid u-u_{0}\right], \quad K_{n}^{++}=K\left[\begin{array}{cc}
n-1 & n-2 \\
n-1 & n
\end{array} \mid u-u_{0}\right] \\
T_{m, n}^{+-}=\operatorname{Tr}_{\mathcal{F}_{m, n}}\left(\Phi(u-1)_{n-1}^{n} \Phi(u)_{n}^{n-1} x^{4 H_{n}}\right), \\
T_{m, n}^{++}=\operatorname{Tr}_{\mathcal{F}_{m, n}}\left(\Phi(u-1)_{n-1}^{n} \Phi(u)_{n-2}^{n-1} \Lambda\left(u_{0}\right)_{n}^{n-2} x^{4 H_{n}}\right) .
\end{gathered}
$$

In Appendix D we perform the direct calculation using the free field representation. The answer is $m$ and $u_{0}$ independent and is given by the well-known Baxter-Kelland formula ${ }^{5}$

$$
\langle\varepsilon\rangle^{(i)}=-(-1)^{i} \frac{\left(x^{2} ; x^{2}\right)_{\infty}^{2}}{\left(-x^{2} ; x^{2}\right)_{\infty}^{2}} \int_{C_{0}} \frac{\epsilon d v}{\mathrm{i} \pi} \frac{\theta_{4}\left(\frac{v-1 / 2}{r} ; \frac{\mathrm{i} \pi}{\epsilon r}\right)}{\theta_{1}\left(\frac{v-1 / 2}{r} ; \frac{\mathrm{i} \pi}{\epsilon r}\right)}=(-)^{i} \frac{\left(x^{2} ; x^{2}\right)_{\infty}^{2}\left(-x^{2 r} ; x^{2 r}\right)_{\infty}^{2}}{\left(-x^{2} ; x^{2}\right)_{\infty}^{2}\left(x^{2 r} ; x^{2 r}\right)_{\infty}^{2}} .
$$

This gives an additional 'finite temperature' check of the correspondence of ground states of the eight-vertex and SOS models.

\section{Discussion}

We proposed a free field construction for correlation functions of the eight-vertex model. The main scheme is the following. We have the bosonic representation for the SOS model, and we know the vertex-face correspondence. Using it we can express objects of the eight-vertex models in terms of the objects in the SOS model. The only nontrivial point is the representation of the operator $\Lambda(u)$ - the unremovable 'tail' of the intertwining vectors. The matrix elements $\Lambda(u)_{n}^{n-2 k}$ with $k \geq 0$ are represented up to a constant factor as powers of Lukyanov's screening operator $X^{k}(u)$, and $\Lambda(u)_{n}^{n+2 k}$ remain undefined. Nevertheless, it is possible to calculate necessary correlation functions using the fact that the SOS model admits of two free field representations. 
Our construction is checked in several ways:

- it supplies solutions to the defining relations for correlation functions;

- it gives the correct spectrum of the corner Hamiltonian;

- it gives the correct average staggered polarization.

Nevertheless there still remain some open problems. The most fundamental problem is why we may substitute traces over the configuration space $\mathcal{H}_{m, n}$ by traces over the Fock $\mathcal{F}_{m, n}$ ? In the case of the six-vertex model the identification of the configuration and Fock spaces is based on the quantum affine symmetry and low temperature expansions. ${ }^{9,25}$ In the case of SOS model there is no such symmetry algebra, which could be defined directly on the lattice. We believe that the solution of this problem could also clarify inconsistency of the involutions in the algebra of vertex operators and in the Heisenberg algebra. This inconsistency forces us to use two free field representations for vertex operators to find correlation functions.

An important problem is to prove $m$ and $u_{0}$ independence of all correlation functions in the framework of the free field representation. This would be a strong argument for the consistency of our approach.

Another group of questions is related with the problem of calculation of form factors. For the six-vertex, SOS, and RSOS models we know that there exist type II vertex operators, that describe asymptotic states in the models, and allow one to obtain general form factors of local operators. The existence of such vertex operators is substantiated by the construction of the elliptic algebra. ${ }^{26}$ To find the representation for the form factors it seems to be necessary to find a free field representation of generic matrix elements $\Lambda\left(u_{0}\right)_{m}^{m^{\prime} n^{\prime}}{ }_{n}$.

\section{Acknowledgments}

We are grateful to A. Antonov, A. Belavin, Vl. Dotsenko, M. Jimbo, S. Lukyanov, and T. Miwa for discussions. We are especially indebted to M. Jimbo for sending us his unpublished notes on the problem. The work was supported in part by the CRDF under the grant RP1-277, and by INTAS and RFBR under the grant INTAS-RFBR-95-690.

\section{Appendix A}

Let us consider a trace like (5.13). The formal series that define this trace is convergent in the region

$$
\left|x^{-4} z_{0}\right|>\left|x^{-2} z\right|>|z|>\left|z_{0}\right| \quad \text { or } \quad \operatorname{Re} u_{0}-2<\operatorname{Re} u-1<\operatorname{Re} u<\operatorname{Re} u_{0} .
$$

So we must consider the trace for arbitrary values of $u$ and $u_{0}$ as an analytic continuation from this region. Besides we know that in the physical region (the regime $I I I$ )

$$
0<u<1
$$

As a consequence of these conditions we have

$$
-1<\operatorname{Re}\left(u-u_{0}\right)<0, \quad-2<\operatorname{Re}\left(-u_{0}\right)<0 .
$$

On the other hand the intertwining vectors are contained in three objects: operator $\Phi_{\varepsilon}\left(u, u_{0}\right)$ contains $t_{\varepsilon}\left(u-u_{0}\right)$; operator $\Phi_{\varepsilon}\left(u-1, u_{0}\right)$ contains $t_{\varepsilon}^{*}\left(u-u_{0}\right)$; operator $\rho\left(u_{0}\right)$ contains $t_{\varepsilon}\left(-u_{0}\right)$ and $t_{\varepsilon}^{*}\left(-u_{0}\right)$. So all the intertwining vectors $t_{\varepsilon}(v)_{n}^{n^{\prime}}, t_{\varepsilon}^{*}(v)_{n}^{n^{\prime}}$ entering into the correlation function representation are of the argument in the region

$$
-2<\operatorname{Re} v<0
$$

\section{Appendix B}

Here we prove the identity

$$
\sum_{n \in 2 \mathbf{Z}+m+i}[n] \chi_{m, n}\left(x^{4}\right)=[m]^{\prime} \chi^{(i)}\left(x^{4}\right)
$$


We have

$$
\begin{aligned}
\sigma^{(i)} & =\sum_{n \in 2 \mathbf{Z}+m+i}[n] \chi_{m, n}\left(x^{4}\right) \\
& =\sum_{n \in 2 \mathbf{Z}+m+i}[n] \frac{x^{2 P_{m, n}^{2}}}{\left(x^{4} ; x^{4}\right)_{\infty}}=\sum_{n \in 2 \mathbf{Z}+m+i} \frac{x^{4 h_{m, n}^{\prime}}}{\left(x^{4} ; x^{4}\right)_{\infty}} \Theta_{x^{2 r}}\left(x^{2 n}\right)
\end{aligned}
$$

with

$$
h_{m, n}^{\prime}=\frac{P_{m, n}^{2}}{2}+\frac{1}{4}\left(\frac{n^{2}}{r}-n\right)=\frac{n^{2}}{4}-\frac{(2 m+1) n}{4}+\frac{1}{4} \frac{r}{r-1} m^{2} .
$$

We see that the fractional coefficient at $n^{2}$ in $2 P_{m, n}^{2}$ is compensated in $4 h_{m, n}^{\prime}$ by the fractional coefficient that takes its origin in $[n]$.

Now let us apply the identity

$$
\Theta_{p}(z)=\sum_{k \in \mathbf{Z}}(-1)^{k} p^{k(k+1) / 2} z^{-k}
$$

We obtain

$$
\begin{aligned}
\sigma^{(i)} & =\frac{1}{\left(x^{4} ; x^{4}\right)_{\infty}} \sum_{k \in \mathbf{Z}}(-1)^{k} x^{r k(k+1)+\frac{r}{r-1} m^{2}} a_{k}^{(i)}, \\
a_{k}^{(i)} & =\sum_{n \in 2 \mathbf{Z}+m+i} x^{n^{2}-(2 m+2 k+1) n} \\
& =x^{-(k+m)(k+m+1)} \sum_{l \in \mathbf{Z}+\frac{1}{2}(k+i)} x^{4 l^{2}-2 l}=x^{-(k+m)(k+m+1)} \sum_{l \in \mathbf{Z}} x^{4 l^{2} \mp 2 l} \\
& =x^{-(k+m)(k+m+1)} \Theta_{x^{8}}\left(-x^{2}\right)=x^{-(k+m)(k+m+1)} \frac{\left(x^{4} ; x^{4}\right)_{\infty}}{\left(x^{2} ; x^{4}\right)_{\infty}} .
\end{aligned}
$$

Here we used the substitution $n \rightarrow 2 l+k+m$ and then $l \rightarrow l+\frac{1}{2}$ for odd $k$. The equality in the last line is easily obtained by use of the evident identities

$$
(z ; p)_{\infty}=\left(z ; p^{2}\right)_{\infty}\left(z p ; p^{2}\right)_{\infty}, \quad\left(z^{2} ; p^{2}\right)_{\infty}=(z ; p)_{\infty}(-z ; p)_{\infty}
$$

So we have

$$
\sigma^{(i)}=\frac{1}{\left(x^{2} ; x^{4}\right)_{\infty}} \sum_{k \in \mathbf{Z}}(-1)^{k} x^{(r-1) k(k+1)-2 m k+\frac{m^{2}}{r-1}-m}=\frac{[m]^{\prime}}{\left(x^{2} ; x^{4}\right)_{\infty}}
$$

q. e. d.

Note that this proof, as well as the consideration in Appendix $\mathrm{D}$, is valid for $m, n \in \mathbf{Z}+\delta$ with any $\delta$.

\section{Appendix C}

In this Appendix we prove that the operators (5.11) satisfy the commutation relation (4.13). Namely, consider the quantity

$$
\begin{aligned}
d\left(u, u_{0}\right)= & \Lambda\left(u_{0}\right)_{n+1}^{n+1-2 k} \Phi(u)_{n}^{n+1}-L\left[\begin{array}{cc}
n+1-2 k & n+2-2 k \\
n+1 & n
\end{array} \mid u_{0}-u\right] \Phi(u)_{n+2-2 k}^{n+1-2 k} \Lambda\left(u_{0}\right)_{n}^{n+2-2 k} \\
& -L\left[\begin{array}{cc}
n+1-2 k & n-2 k \\
n+1 & n
\end{array} \mid u_{0}-u\right] \Phi(u)_{n-2 k}^{n+1-2 k} \Lambda\left(u_{0}\right)_{n}^{n-2 k}
\end{aligned}
$$


We have to prove that $d\left(u, u_{0}\right)=0$ from the free field representation inputs. Substituting Eq. (5.5) we obtain

$$
\begin{aligned}
d\left(u, u_{0}\right) \sim & {[n+1-2 k] X^{k}\left(u_{0}\right) V(u)+(-1)^{k} \frac{\left[u_{0}-u+n+1-k\right][k]}{\left[u_{0}-u\right]} V(u) X(u) X^{k-1}\left(u_{0}\right) } \\
& -(-1)^{k} \frac{\left[u_{0}-u+k\right][n+1-k]}{\left[u_{0}-u\right]} V(u) X^{k}\left(u_{0}\right) .
\end{aligned}
$$

Here the sign $\sim$ only means that two functions differ by a factor which is allowed to be any nonzero function of all variables. Substituting Eq. (5.4) and taking into account that

$$
V\left(u_{1}\right) \bar{V}\left(u_{2}\right)=-\frac{\left[u_{1}-u_{2}+\frac{1}{2}\right]}{\left[u_{1}-u_{2}-\frac{1}{2}\right]} \bar{V}\left(u_{2}\right) V\left(u_{1}\right)
$$

we obtain

$$
d\left(u, u_{0}\right) \sim \int \frac{d^{k} v}{(2 \pi \mathrm{i})^{k}} V(u) \bar{V}\left(v_{k}\right) \ldots \bar{V}\left(v_{1}\right) f\left(u, u_{0} ; v_{1}, \ldots, v_{k}\right)
$$

with

$$
\begin{aligned}
f\left(u, u_{0} ; v_{1}, \ldots, v_{k}\right)= & \operatorname{Sym}\left\{[n+1-2 k] \prod_{i=1}^{k} \frac{\left[v_{i}-u_{0}+\frac{1}{2}-n-1+2(i-1)\right]}{\left[v_{i}-u_{0}-\frac{1}{2}\right]} \frac{\left[v_{i}-u+\frac{1}{2}\right]}{\left[v_{i}-u-\frac{1}{2}\right]}\right. \\
& +\frac{\left[u_{0}-u+n+1-k\right][k]}{\left[u_{0}-u\right]} \frac{\left[v_{k}-u-n+2 k-\frac{3}{2}\right]}{\left[v_{k}-u-\frac{1}{2}\right]} \prod_{i=1}^{k-1} \frac{\left[v_{i}-u_{0}+\frac{1}{2}-n+2(i-1)\right]}{\left[v_{i}-u_{0}-\frac{1}{2}\right]} \\
& \left.-\frac{\left[u_{0}-u+k\right][n+1-k]}{\left[u_{0}-u\right]} \prod_{i=1}^{k} \frac{\left[v_{i}-u_{0}+\frac{1}{2}-n+2(i-1)\right]}{\left[v_{i}-u_{0}-\frac{1}{2}\right]}\right\} .
\end{aligned}
$$

Here the operation Sym is defined as

$$
\operatorname{Sym} F\left(v_{1}, \ldots, v_{k}\right)=\frac{1}{k !} \sum_{\sigma \in S_{k}} F\left(v_{\sigma(1)}, \ldots, v_{\sigma(k)}\right) \prod_{\substack{i<j \\ \sigma(i)>\sigma(j)}} h\left(v_{\sigma(i)}-v_{\sigma(j)}\right)
$$

with $h(v)$ being defined as

$$
\bar{V}\left(v_{1}\right) \bar{V}\left(v_{2}\right)=h\left(v_{1}-v_{2}\right) \bar{V}\left(v_{2}\right) \bar{V}\left(v_{1}\right), \quad h(v)=\frac{[v-1]}{[v+1]} .
$$

This 'symmetrization' of the function $f$ corresponds to symmetrization of the integrand.

So we have to prove that $f\left(u, u_{0} ; v_{1}, \ldots, v_{k}\right)=0$.

To calculate $f$ let us use the identity ${ }^{27}$

$$
\operatorname{Sym} \prod_{i=1}^{k}\left[v_{i}-2 i+2\right]=\frac{[k] !}{k ![1]^{k}} \prod_{i<j} \frac{\left[v_{i}-v_{j}\right]}{\left[v_{i}-v_{j}-1\right]} \prod_{i=1}^{k}\left[v_{i}-k+1\right]
$$

with $[k] !=\prod_{i=1}^{k}[i]$. We apply this function directly to the first and last terms, but in the second term we only apply it to $k-1$ variables, and symmetrize in the remaining variable by hand. We have

$$
f\left(u, u_{0} ; v_{1}, \ldots, v_{N}\right) \sim g(u)
$$

with $g(u)$ being a doubly periodic function of $u$ (and all other variables):

$$
\begin{aligned}
g(u)= & 1-\frac{[n+1-2 k]}{[n-k+1]} \frac{\left[u_{0}-u\right]}{\left[u_{0}-u+k\right]} \prod_{i=1}^{k} \frac{\left[v_{i}-u_{0}-n+k-\frac{3}{2}\right]\left[v_{i}-u+\frac{1}{2}\right]}{\left[v_{i}-u_{0}-n+k-\frac{1}{2}\right]\left[v_{i}-u-\frac{1}{2}\right]} \\
& -\frac{\left[u_{0}-u+n+1-k\right][1]}{\left[u_{0}-u+k\right][n-k+1]} \prod_{i=1}^{k} \frac{\left[v_{i}-u_{0}-n+k-\frac{3}{2}\right]}{\left[v_{i}-u_{0}-n+k-\frac{1}{2}\right]} \\
& \times \sum_{j=1}^{k}\left(\frac{\left[v_{j}-u-n+2 k-\frac{3}{2}\right]\left[v_{j}-u_{0}-\frac{1}{2}\right]}{\left[v_{j}-u-\frac{1}{2}\right]\left[v_{j}-u_{0}-n+k-\frac{3}{2}\right]} \prod_{i \neq j} \frac{\left[v_{i}-v_{j}+1\right]}{\left[v_{i}-v_{j}\right]}\right) .
\end{aligned}
$$


It is easy to check that

$$
g\left(u_{0}+n+1-k\right)=0 .
$$

So it is enough to prove that the function $g\left(u_{0}\right)$ is constant. It has poles at the points (up to periods)

$$
\begin{aligned}
& u=u_{0}+k, \\
& u=v_{l}-1 / 2 .
\end{aligned}
$$

It is straightforward to check that the residue at $u=v_{l}-\frac{1}{2}$ vanishes. For the residue at $u=u_{0}+k$ we have

$$
\operatorname{Res}_{u=u_{0}+k} g(u) \sim g_{0}\left(u_{0}\right)=1-\frac{1}{[k]} \prod_{i=1}^{k} \frac{\left[v_{i}-u_{0}-k-\frac{1}{2}\right]}{\left[v_{i}-u_{0}-k+\frac{1}{2}\right]} \sum_{j=1}^{k}\left(\frac{\left[v_{j}-u_{0}-\frac{1}{2}\right]}{\left[v_{j}-u_{0}-k-\frac{1}{2}\right]} \prod_{i \neq j} \frac{\left[v_{i}-v_{j}+1\right]}{\left[v_{i}-v_{j}\right]}\right) .
$$

It is easy to check that $g_{0}\left(v_{l}-k-\frac{1}{2}\right)=0$. For the residues we have

$$
\operatorname{Res}_{u_{0}=v_{l(1)}-k+\frac{1}{2}} g_{0}\left(u_{0}\right) \sim g_{1}\left(v_{l(1)}\right)=1-\frac{1}{[k-1]} \sum_{j \neq l(1)}\left(\frac{\left[v_{j}-v_{l(1)}+k-1\right]}{\left[v_{j}-v_{l(1)}+1\right]} \prod_{i \neq j, l(1)} \frac{\left[v_{j}-v_{i}-1\right]\left[v_{l(1)}-v_{i}\right]}{\left[v_{j}-v_{i}\right]\left[v_{l(1)}-v_{i}-1\right]}\right) .
$$

To proceed by induction introduce the functions

$$
g_{s}\left(v_{l(s)}\right)=1-\frac{1}{[k-s]} \sum_{j \neq l(1), \ldots, l(s)}\left(\prod_{p=1}^{s} \frac{\left[v_{j}-v_{l(p)}+k-1\right]}{\left[v_{j}-v_{l(p)}+1\right]} \prod_{i \neq j, l(1), \ldots, l(s)} \prod_{p=1}^{s} \frac{\left[v_{j}-v_{i}-1\right]\left[v_{l(p)}-v_{i}\right]}{\left[v_{j}-v_{i}\right]\left[v_{l(p)}-v_{i}-1\right]}\right) .
$$

We suppose that $l(1), \ldots, l(k)$ is an arbitrary permutation of the numbers $1, \ldots, k\left(\right.$ i. e. $\left.l \in S_{k}\right)$. We have

$$
g_{s}\left(v_{l(s+1)}\right)=0, \quad \operatorname{Res}_{v_{l(s)}=v_{l(s+1)}+1} g_{s}\left(v_{l(s)}\right) \sim g_{s+1}\left(v_{l(s+1)}\right), \quad g_{k}\left(v_{l(k)}\right)=0 .
$$

Hence it follows that all $g_{s}$ and $f$ vanish. This completes the proof in the case $s=n+1$. The proof in the case $s=n-1$ is similar though more cumbersome.

\section{Appendix D}

Here we show that the free field representation for the eight-vertex model results in the Baxter-Kelland formula for the staggered polarization.

As the initial point of the calculation we take Eq. (5.16). For the coefficients $K$ we have

$$
\begin{aligned}
& K\left[\begin{array}{cc}
n^{\prime} & n^{\prime} \pm 1 \\
n & n \pm 1
\end{array} \mid u\right]=\frac{h_{4}\left(u \pm \frac{n-n^{\prime}}{2}\right) h_{4}\left(\frac{n+n^{\prime}}{2}\right)}{h_{1}(u)}, \\
& K\left[\begin{array}{cc}
n^{\prime} & n^{\prime} \mp 1 \\
n & n \pm 1
\end{array} \mid u\right]=\frac{h_{4}\left(u \pm \frac{n+n^{\prime}}{2}\right) h_{4}\left(\frac{n-n^{\prime}}{2}\right)}{h_{1}(u)}
\end{aligned}
$$

for $n^{\prime}-n \in 2 \mathbf{Z}$, with

$$
h_{i}(u)=\sqrt{\frac{\pi}{\epsilon r}} \mathrm{e}^{\frac{1}{4} \epsilon r} \theta_{i}\left(\frac{u}{r} ; \frac{\mathrm{i} \pi}{\epsilon r}\right), \quad h_{1}(u)=[u], \quad h_{4}(u)=\mathrm{ie}^{-\frac{\pi^{2}}{4 r \epsilon}-\mathrm{i} \pi \frac{u}{r}}[u-\mathrm{i} \pi / 2 \epsilon] .
$$

Hence

$$
\begin{aligned}
K_{n}^{+-} & =\frac{h_{4}(n-1) h_{4}(u)}{h_{1}(u)}, \\
K_{n}^{++} & =\frac{h_{4}(n-1+u) h_{4}(0)}{h_{1}(u)} .
\end{aligned}
$$


The traces $T_{m, n}^{+-}$and $T_{m, n}^{++}$can be expressed as follows

$$
T_{m, n}^{+-}=-\frac{\mathrm{i}}{[n-1][n]} T_{m, n}(u, u), \quad T_{m, n}^{++}=\frac{\mathrm{i}}{[n-1][n]} T_{m, n}\left(u, u_{0}\right)
$$

with

$$
\begin{aligned}
T_{m, n}\left(u, u_{0}\right) & =\operatorname{Tr}_{\mathcal{F}_{m, n}}\left(V(u-1) V(u) X\left(u_{0}\right) x^{4 H}\right) \\
& =\epsilon \int \frac{d v}{\mathrm{i} \pi} H_{m, n}\left(u, u_{0} ; v\right) G(v-u) .
\end{aligned}
$$

Here $G(v)$ is the contribution into the trace in the second line from the oscillators $a_{n}$ :

$$
\begin{aligned}
G(v) & =\frac{\eta^{-1} \mathcal{N}}{\left(x^{4} ; x^{4}\right)_{\infty}} \frac{\left(x^{2} ; x^{2}\right)_{\infty}}{\left(x^{2 r} ; x^{2 r}\right)_{\infty}} \frac{\Theta_{x^{2 r}}\left(x^{-1} \zeta\right)}{\Theta_{x^{2}}\left(x^{-1} \zeta\right)} \\
& =\mathrm{i} \frac{\left(x^{2} ; x^{2}\right)_{\infty}^{2}}{\left(-x^{2} ; x^{2}\right)_{\infty}} \mathrm{e}^{\epsilon \frac{r-1}{2 r}-\epsilon \frac{r-1}{r}\left(v-\frac{1}{2}\right)^{2}} \frac{h_{1}\left(v-\frac{1}{2}\right)}{\vartheta_{1}\left(v-\frac{1}{2}\right)}, \quad \zeta=x^{2 v}, \\
\eta^{-1} \mathcal{N} & =\mathrm{i} x^{-\frac{r-1}{2 r}}\left(x^{2} ; x^{2}\right)_{\infty}^{2}\left(x^{2 r} ; x^{2 r}\right)_{\infty}, \quad \vartheta_{i}(u)=\sqrt{\frac{\pi}{\epsilon}} \mathrm{e}^{\frac{1}{4} \epsilon} \theta_{i}(u ; \mathrm{i} \pi / \epsilon) .
\end{aligned}
$$

The factor $\mathcal{N}$ originates from the traces of individual exponential operators, whereas the rest originates from traces of pair products in the generalized Wick theorem.

The factor $H_{m, n}\left(u, u_{0} ; v\right)$ denotes the contribution of the 'zero mode':

$$
\begin{aligned}
H_{m, n}\left(u, u_{0} ; v\right)= & \left\langle P_{m, n}\right| x^{2 \mathcal{P}^{2}} \mathrm{e}^{-\mathrm{i} \sqrt{\frac{r-1}{2 r}} \mathcal{Q}}\left(x^{-2} z\right)^{-\sqrt{\frac{r-1}{2 r}} \mathcal{P}+\frac{r-1}{4 r}} \mathrm{e}^{-\mathrm{i} \sqrt{\frac{r-1}{2 r}} \mathcal{Q}} z^{-\sqrt{\frac{r-1}{2 r}} \mathcal{P}+\frac{r-1}{4 r}} \\
& \times \mathrm{e}^{\mathrm{i} \sqrt{2 \frac{r-1}{r}} \mathcal{Q}} \zeta^{\sqrt{2 \frac{r-1}{r}} \mathcal{P}+\frac{r-1}{r}} \frac{\left[v-u_{0}+1 / 2-\sqrt{2 r(r-1)} \mathcal{P}\right]}{\left[v-u_{0}-1 / 2\right]}\left|P_{m, n}\right\rangle \\
= & (-)^{n-m} \mathrm{e}^{-2 \epsilon \frac{r-1}{r}\left(v-u+\frac{1}{4}\right)-2 \epsilon\left(v-u+\frac{1}{2}\right) m-\epsilon \frac{r}{r-1} m^{2}-\epsilon \frac{r-1}{r} n^{2}+2 \epsilon m n+2 \epsilon\left(v-u+\frac{1}{2}\right) n} \\
& \times \frac{h_{1}\left(v-u_{0}+\frac{1}{2}-n\right)}{h_{1}\left(v-u_{0}-\frac{1}{2}\right)} .
\end{aligned}
$$

It is convenient to calculate the quantity

$$
\begin{aligned}
H_{m}^{(i)}\left(u, u_{0} ; v\right) & =\sum_{n \in 2 \mathbf{Z}+m+i} \frac{1}{[n-1]}\left(K_{n}^{++} H_{m, n}\left(u, u_{0} ; v\right)-K_{n}^{+-} H_{m, n}(u, u ; v)\right) \\
= & (-)^{i} \sum_{n} \frac{\mathrm{e}^{-2 \epsilon \frac{r-1}{r}\left(v-u+\frac{1}{4}\right)-2 \epsilon\left(v-u+\frac{1}{2}\right) m-\epsilon \frac{r}{r-1} m^{2}-\epsilon \frac{r-1}{r} n^{2}-2 \epsilon m n+2 \epsilon \frac{r-1}{r}\left(v-u+\frac{1}{2}\right) n}}{h_{1}\left(u-u_{0}\right) h_{1}(n-1) h_{1}\left(v-u-\frac{1}{2}\right) h_{1}\left(v-u_{0}-\frac{1}{2}\right)} \\
& \times\left\{h_{1}\left(v-u-\frac{1}{2}\right) h_{1}\left(v-u_{0}+\frac{1}{2}-n\right) h_{4}\left(n-1+u-u_{0}\right) h_{4}(0)\right. \\
& \left.-h_{1}\left(v-u_{0}-\frac{1}{2}\right) h_{1}\left(v-u+\frac{1}{2}-n\right) h_{4}(n-1) h_{4}\left(u-u_{0}\right)\right\} .
\end{aligned}
$$

Substituting the identity

$$
\begin{aligned}
& \theta_{4}(2 x) \theta_{4}(2 y) \theta_{1}(2 z) \theta_{1}(2 t) \\
& \quad=\theta_{4}(x+y+z+t) \theta_{4}(x+y-z-t) \theta_{1}(x-y-z+t) \theta_{1}(x-y+z-t) \\
& \quad+\theta_{4}(x+y+z-t) \theta_{4}(x+y-z+t) \theta_{1}(x-y+z+t) \theta_{1}(-x+y+z+t),
\end{aligned}
$$

we obtain

$$
\begin{aligned}
H_{m}^{(i)}\left(u, u_{0} ; v\right)= & (-)^{i} \sum_{n} \mathrm{e}^{-2 \epsilon \frac{r-1}{r}\left(v-u+\frac{1}{4}\right)-2 \epsilon\left(v-u+\frac{1}{2}\right) m-\epsilon \frac{r}{r-1} m^{2}-\epsilon \frac{r-1}{r} n^{2}+2 \epsilon m n+2 \epsilon \frac{r-1}{r}\left(v-u+\frac{1}{2}\right) n} \\
& \times \frac{h_{4}\left(v-u_{0}-\frac{1}{2}\right) h_{4}\left(v-u+\frac{1}{2}-n\right)}{h_{1}\left(v-u_{0}-\frac{1}{2}\right) h_{1}\left(v-u-\frac{1}{2}\right)} .
\end{aligned}
$$


From the summand we can extract an $n$-dependent factor

$h_{m, n}^{(i)}=\mathrm{e}^{-\epsilon \frac{r-1}{r} n^{2}+2 \epsilon m n+2 \epsilon \frac{r-1}{r} w n} \theta_{4}\left(\frac{w-n}{r} ; \frac{\mathrm{i} \pi}{\epsilon r}\right)=\sqrt{\frac{\epsilon r}{\pi}} \mathrm{e}^{-\epsilon \frac{r-1}{r} n^{2}+2 \epsilon m n+2 \epsilon \frac{r-1}{r} w n-\frac{\epsilon}{r}(w-n)^{2}} \theta_{2}\left(\mathrm{i} \frac{\epsilon}{\pi}(w-n) ; \mathrm{i} \frac{\epsilon r}{\pi}\right)$

with $w=v-u+\frac{1}{2}$. Expanding the last theta function in a series, we obtain

$$
\sum_{n \in 2 \mathbf{Z}+m+i} h_{m, n}^{(i)}=\sqrt{\frac{\epsilon r}{\pi}} \sum_{p \in \mathbf{Z}+\frac{1}{2}(m+i)} \sum_{s \in \mathbf{Z}+\frac{1}{2}} \mathrm{e}^{-\epsilon r s^{2}-2 \epsilon w s-4 \epsilon p^{2}+4 \epsilon m p+4 \epsilon w p+4 \epsilon s p-\frac{\epsilon}{r} w^{2}}
$$

Now we can split the sum into two terms: the first with even $s-m-\frac{1}{2}$ and the second with even $s-m+\frac{1}{2}$.

Each term factors after change of summation variables $(p, s)$ to $(k, l)$ so that $s=m \pm \frac{1}{2}+2 l$ and $p=k+l$.

Each sum in $k$ and $l$ gives a theta function.

$$
\begin{aligned}
H_{m}^{(i)}\left(u, u_{0} ; v\right)= & (-)^{i} \mathrm{e}^{\frac{1}{4} \epsilon r-2 \epsilon \frac{r-1}{r}\left(v-u+\frac{1}{4}\right)+\epsilon \frac{r-1}{r}\left(v-u+\frac{1}{2}\right)^{2}} \frac{h_{4}\left(v-u_{0}-\frac{1}{2}\right)}{h_{1}\left(v-u_{0}-\frac{1}{2}\right) h_{1}\left(v-u-\frac{1}{2}\right)} \\
& \times\left\{\mathrm{e}^{-\frac{\epsilon}{r-1}\left(r m-\frac{r-1}{2}\right)^{2}-\epsilon\left(w+\frac{1}{2}\right)^{2}} \theta_{3}\left(\mathrm{i} \frac{2 \epsilon}{\pi}\left(r m-\frac{r-1}{2}\right) ; \mathrm{i} \frac{4 \epsilon(r-1)}{\pi}\right) \theta_{3,2}\left(\mathrm{i} \frac{2 \epsilon}{\pi}\left(w+\frac{1}{2}\right) ; \mathrm{i} \frac{4 \epsilon}{\pi}\right)\right. \\
& \left.+\mathrm{e}^{-\frac{\epsilon}{r-1}\left(r m+\frac{r-1}{2}\right)^{2}-\epsilon\left(w-\frac{1}{2}\right)^{2}} \theta_{3}\left(\mathrm{i} \frac{2 \epsilon}{\pi}\left(r m+\frac{r-1}{2}\right) ; \mathrm{i} \frac{4 \epsilon(r-1)}{\pi}\right) \theta_{3,2}\left(\mathrm{i} \frac{2 \epsilon}{\pi}\left(w-\frac{1}{2}\right) ; \mathrm{i} \frac{4 \epsilon}{\pi}\right)\right\} .
\end{aligned}
$$

Here the subscript 3 or 2 depends on evenness of $m+i$. Making the module conjugation transformation we get

$$
\begin{aligned}
H_{m}^{(i)}\left(u, u_{0} ; v\right)= & \frac{(-1)^{i}}{4 \sqrt{r-1}} \mathrm{e}^{\frac{1}{4} \epsilon r-2 \epsilon \frac{r-1}{r}\left(v-u+\frac{1}{4}\right)+\epsilon \frac{r-1}{r}\left(v-u+\frac{1}{2}\right)^{2}} \frac{h_{4}\left(v-u_{0}-\frac{1}{2}\right)}{h_{1}\left(v-u_{0}-\frac{1}{2}\right) h_{1}\left(v-u-\frac{1}{2}\right)} \\
& \times\left\{\theta_{3}\left(\frac{1}{2} \frac{r}{r-1} m-\frac{1}{4} ; \frac{\mathrm{i} \pi}{4 \epsilon(r-1)}\right) \theta_{3,4}\left(\frac{w}{2}+\frac{1}{4} ; \frac{\mathrm{i} \pi}{4 \epsilon}\right)\right. \\
& \left.+\theta_{3}\left(\frac{1}{2} \frac{r}{r-1} m+\frac{1}{4} ; \frac{\mathrm{i} \pi}{4 \epsilon(r-1)}\right) \theta_{3,4}\left(\frac{w}{2}-\frac{1}{4} ; \frac{\mathrm{i} \pi}{4 \epsilon}\right)\right\} .
\end{aligned}
$$

Here the subscript 3 of the second and forth theta functions corresponds to even $m+i$ and the index 4 to odd one. In principle, the above resummation procedure is the same as that of the Appendix B.

Calculating $H_{m}^{(i)}-H_{-m}^{(i)}$ it is convenient to use the identity

$$
\theta_{3}\left(u+\frac{1}{4} ; \tau\right)-\theta_{3}\left(u-\frac{1}{4} ; \tau\right)=-2 \theta_{1}(2 u ; 4 \tau)
$$

Applying it twice, we get

$$
H_{m}^{(i)}-H_{-m}^{(i)}=\mathrm{e}^{-2 \epsilon \frac{r-1}{r}\left(v-u+\frac{1}{4}\right)+\epsilon \frac{r-1}{r}\left(v-u+\frac{1}{2}\right)^{2}} \frac{h_{4}\left(v-u_{0}-\frac{1}{2}\right) \vartheta_{1}\left(v-u-\frac{1}{2}\right)}{h_{1}\left(v-u_{0}-\frac{1}{2}\right) h_{1}\left(v-u-\frac{1}{2}\right)}[m]^{\prime} .
$$

The factor $[m]^{\prime}$ here will cancel the respective quantity in the denominator in Eq. (5.16). The $u$ depending theta functions here will cancel theta functions in $G(v)$.

Gathering all results we obtain

$$
\begin{aligned}
\langle\varepsilon\rangle^{(i)} & =\mathrm{i}(-1)^{i} \frac{\left(x^{2} ; x^{4}\right)_{\infty}}{[m]^{\prime}} \epsilon \int \frac{d v}{\mathrm{i} \pi}\left(H_{m}^{(i)}\left(u, u_{0} ; v\right)-H_{-m}^{(i)}\left(u, u_{0} ; v\right)\right) G(v-u) \\
& =-(-1)^{i} \frac{\left(x^{2} ; x^{2}\right)_{\infty}^{2}}{\left(-x^{2} ; x^{2}\right)_{\infty}^{2}} I
\end{aligned}
$$


where

$$
I=\epsilon \int_{C_{0}} \frac{d v}{\mathrm{i} \pi} \frac{\theta_{4}\left(\frac{v-1 / 2}{r} ; \frac{\mathrm{i} \pi}{\epsilon r}\right)}{\theta_{1}\left(\frac{v-1 / 2}{r} ; \frac{\mathrm{i} \pi}{\epsilon r}\right)} .
$$

To calculate the integral note that

$$
\theta_{1}(u+1)=-\theta_{1}(u), \quad \theta_{4}(u+1)=\theta_{4}(u)
$$

and

$$
I=-\epsilon \int_{C_{0}} \frac{d v}{\mathrm{i} \pi} \frac{\theta_{4}\left(\frac{v+r-1 / 2}{r} ; \frac{\mathrm{i} \pi}{\epsilon r}\right)}{\theta_{1}\left(\frac{v+r-1 / 2}{r} ; \frac{\mathrm{i} \pi}{\epsilon r}\right)}=-\epsilon \int_{C_{r}} \frac{d v}{\mathrm{i} \pi} \frac{\theta_{4}\left(\frac{v-1 / 2}{r} ; \frac{\mathrm{i} \pi}{\epsilon r}\right)}{\theta_{1}\left(\frac{v-1 / 2}{r} ; \frac{\mathrm{i} \pi}{\epsilon r}\right)} .
$$

But the sum of (D.13) and (D.12) is an integral over a closed path enclosing the pole $v=1 / 2$. Hence

$$
I=-\frac{\epsilon}{2} \oint \frac{d v}{\mathrm{i} \pi} \frac{\theta_{4}\left(\frac{v-1 / 2}{r} ; \frac{\mathrm{i} \pi}{\epsilon r}\right)}{\theta_{1}\left(\frac{v-1 / 2}{r} ; \frac{\mathrm{i} \pi}{\epsilon r}\right)}=-\epsilon \underset{v=1 / 2}{\operatorname{Res}} \frac{\theta_{4}\left(\frac{v-1 / 2}{r} ; \frac{\mathrm{i} \pi}{\epsilon r}\right)}{\theta_{1}\left(\frac{v-1 / 2}{r} ; \frac{\mathrm{i} \pi}{\epsilon r}\right)}=-\epsilon r \frac{\theta_{4}\left(0 ; \frac{\mathrm{i} \pi}{\epsilon r}\right)}{\theta_{1}^{\prime}\left(0 ; \frac{\mathrm{i} \pi}{\epsilon r}\right)} .
$$

Using the multiplicative form of the theta functions we easily obtain

$$
I=-\frac{\left(-x^{2 r} ; x^{2 r}\right)_{\infty}^{2}}{\left(x^{2 r} ; x^{2 r}\right)_{\infty}^{2}}
$$

Substituting (D.14) into (D.11) we obtain the Baxter-Kelland formula (5.17).

\section{References}

1. B. Sutherland, J. Math. Phys. 11, 3183 (1970)

2. C. Fan and F. Y. Wu, Phys. Rev. B2, 723 (1970)

3. R. J. Baxter, Exactly Solved Models in Statistical Mechanics, Academic Press, 1982

4. R. J. Baxter, Phys. Rev. Lett. 26, 832 (1971); Ann. Phys. (N. Y.) 70, 193 (1972)

5. R. J. Baxter and S. B. Kelland, J. Phys. C7, L403 (1974)

6. M. N. Barber and R. J. Baxter, J. Phys. C6, 2913 (1973)

7. R. J. Baxter, Phil. Trans. Royal Soc. London 289, 315 (1978)

8. M. Jimbo, T. Miwa, and A. Nakayashiki, J. Phys. A26 2199 (1993)

9. B. Davies, O. Foda, M. Jimbo, T. Miwa, and A. Nakayashiki, Commun. Math. Phys. 151, 89 (1993)

10. M. Jimbo, K. Miki, T. Miwa, and A. Nakayashiki, Phys. Lett. A168, 256 (1992)

11. M. Jimbo and T. Miwa, Algebraic Analysis of Solvable Lattice Models, CBMS Regional Conference Series in Mathematics, 85, AMS, 1994

12. S. Lukyanov, Commun. Math. Phys. 167, 183 (1995)

13. S. Lukyanov, Phys. Lett. B325, 409 (1994)

14. O. Foda, M. Jimbo, T. Miwa, K. Miki, and A. Nakayashiki, J. Math. Phys. 35, 13 (1994)

15. S. Lukyanov and Ya. Pugai, J. Exp. Theor. Phys. 82, 1021 (1996) (hep-th/9412128)

16. S. Lukyanov and Ya. Pugai, Nucl. Phys. B[FS]473, 631 (1996) (hep-th/9602074)

17. Y. Asai, M. Jimbo, T. Miwa, and Ya. Pugai, J. Phys. A29, 6595 (1996)

18. B. Feigin, M. Jimbo, T. Miwa, A. Odesskii, and Ya. Pugai, Algebra of screening operators for the deformed $W_{n}$ algebra, preprint q-alg/9702029, (February 1997)

19. A. H. Bougourzi, Bosonization of quantum affine groups and its application to the higher spin Heisenberg model, preprint ITP-SB-97-29, q-alg/9706015 (June 1997)

20. H. Konno, An elliptic algebra $U_{q, p}\left(\widehat{s l}_{2}\right)$ and the fusion RSOS model, preprint q-alg/9709013 (September 1997) 
21. J. R. Reyes Martinez, Correlation functions for the Z-invariant Ising model, hep-th/9609135 (September 1996)

22. R. J. Baxter, Ann. Phys. (N. Y.) 76, 1, 25, 48 (1973)

23. M. Jimbo and T. Miwa, unpublished, 1993; E. Frenkel, S. Lukyanov, and N. Reshetikhin, unpublished, 1996; B. Feigin and A. Odesskii, unpublished, 1996; M. Lashkevich and Ya. Pugai, unpublished, 1996; H. Fau, B. Hou, K. Shi, and W. Yang, J. Phys. A30, 5687 (1997)

24. M. Yu. Lashkevich, Mod. Phys. Lett. B10, 101 (1996) (hep-th/9408131)

25. S.-J. Kang, M. Kashiwara, K. C. Misra, T. Miwa, T. Nakashima, and A. Nakayashiki, C. R. Acad. Sci. Paris, 315I, 375 (1992)

26. O. Foda, K. Iohara, M. Jimbo, R. Kedem, T. Miwa, and H. Yan, Lett. Math. Phys. 32, 258 (1994)

27. M. Jimbo, M. Lashkevich, T. Miwa, Ya. Pugai, Phys. Lett. A229, 285 (1997) (hep-th/9607177) 